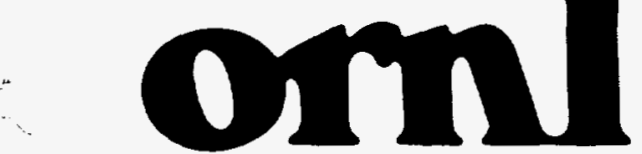

OAK RIDGE NATIONAL LABORATORY

MARTIN MARUETRA

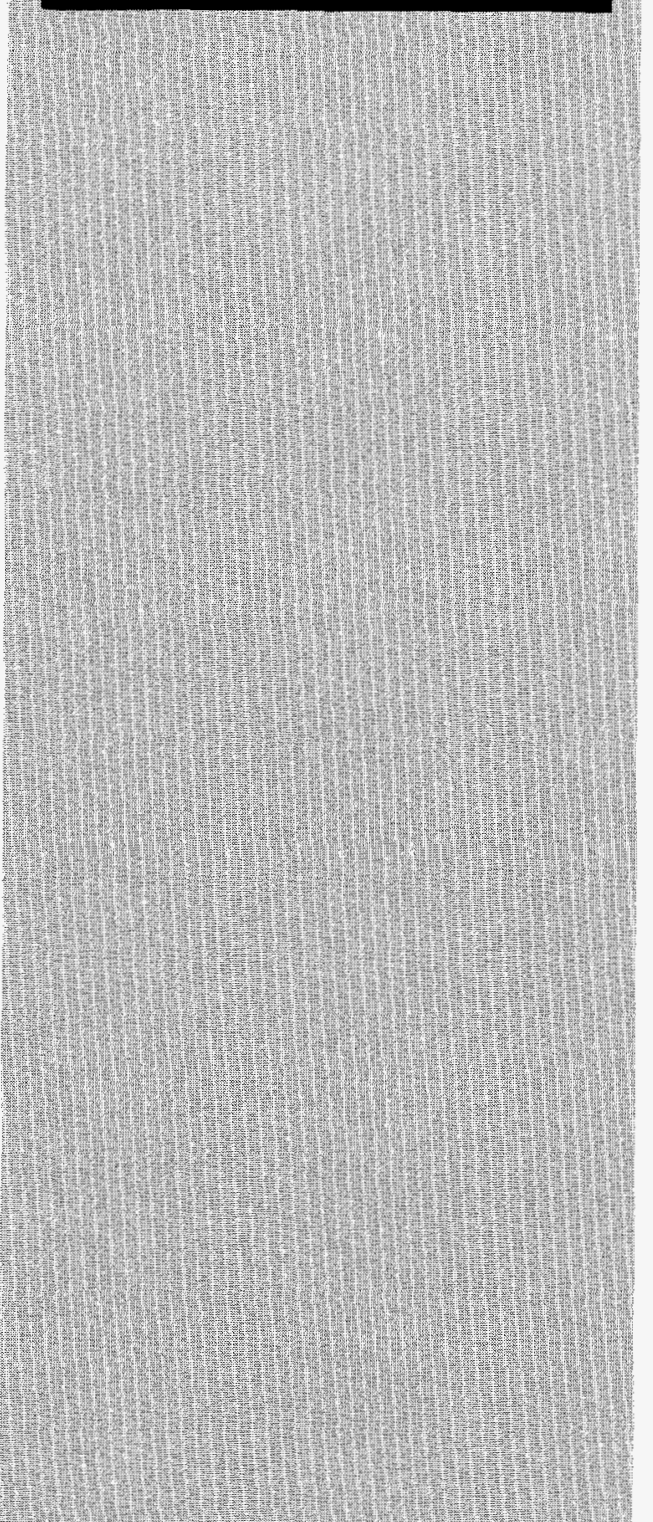

\section{MANAGED BY}

MARTIN MARIETTA ENERGY SYSTEUS, INC. FOR THE UNITEO STATES DEPARTMENT OF ENERGY
Third-Order-Accurate Numerical Methods for Efficient, Large Time-Step Solutions of Mixed Linear and Nonlinear Problems

J. W. Cobb 
This report has been reproduced directly from the best available copy.

Available to DOE and DOE contractors from the Office of Scientific and Technical Information, P.O. Box 62, Oak Ridge, TN 37831; prices available from (615) 576-8401, FTS 626-8401.

Available to the public from the National Technical Information Service, U.S. Department of Commerce, 5285 Port Royal Rd., Springfield, VA 22161.

This report was prepared as an account of work sponsored by an agency of the United States Government. Neither the United States Government nor any agency thereof, nor any of their employees, makes any warranty, express or implied, or assumes any legal liability or responsibility for the accuracy, completeness, or usefulness of any information, apparatus, product, or process disclosed, or represents that its use would not infringe privately owned rights. Reference herein to any specific commercial product, process, or service by trade name, trademark, manufacturer, or otherwise, does not necessarily constitute or imply its endorsement, recommendation, or favoring by the United States Government or any agency thereof. The views and opinions of authors expressed herein do not necessarily state or refiect those of the United States Government or any agency thereof. 


\section{DISCLAIMER}

Portions of this document may be illegible in electronic image products. Images are produced from the best available original document. 
ORNL/TM-12891

Dist. Category UC-427

Fusion Energy Division

\title{
Third-Order-Accurate Numerical Methods for Efficient, Large Time-Step Solutions of Mixed Linear and Nonlinear Problems
}

J. W. Cobb

DATE PUBLISHED - February 1995

Prepared for the

Office of Fusion Energy

Budget Activity AT 0520210

\author{
Prepared by \\ OAK RIDGE NATIONAL LABORATORY \\ Oak Ridge, Tennessee 37831-6285 \\ managed by \\ MARTIN MARIETTA ENERGY SYSTEMS, INC. \\ for the \\ U.S. DEPARTMENT OF ENERGY \\ under contract DE-AC05-84OR21400
}

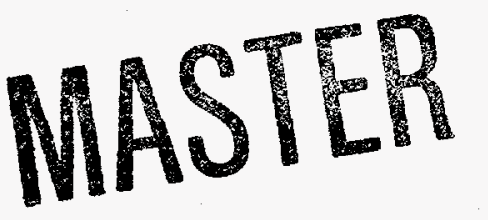





\section{CONTENTS}

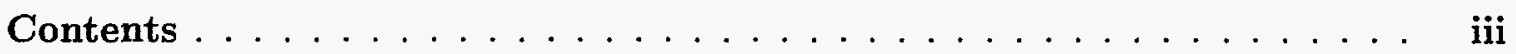

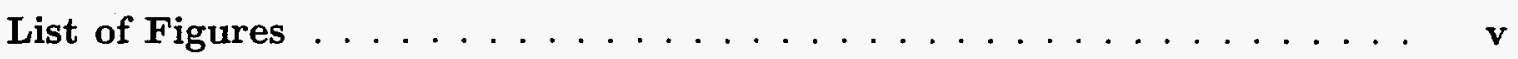

Abstract ........................ vii

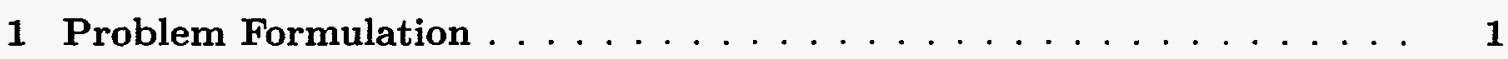

1.1 General Motivation . . . . . . . . . . . . . . . . . . . . . . . . . . . . . . . . .

1.2 Large Time-Steps . . . . . . . . . . . . . . . . . . . . 2

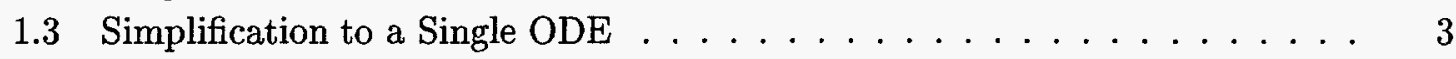

1.4 Requirements on Numerical Method . . . . . . . . . . . . . 3

2 Third-Order Runge-Kutta (RK) Methods . . . . . . . . . . . . 4

2.1 Description of RK Methods . . . . . . . . . . . . . 4

2.2 The Structure of Coefficient Matrices . . . . . . . . . . . . . 5

2.3 General Considerations of the Coefficient Equations . . . . . . . . . 5

2.4 Explicit Construction of Coefficient Equations . . . . . . . . . . . . . 6

2.5 General Parameterized Solution . . . . . . . . . . . . . . . . . . 11

2.6 Equal Implicitness Parameterization . . . . . . . . . . . . . . . . . 12

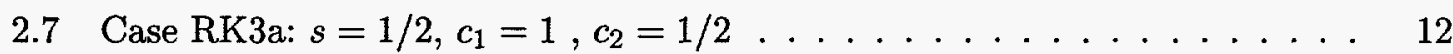

2.8 Case RK3b: $s=1 / 2, c_{1}=1 / 3, c_{2}=2 / 3 \ldots \ldots \ldots \ldots \ldots$

2.9 Case RK3c: $s=1 / 3, c_{1}=1 / 3, c_{2}=2 / 3 \ldots \ldots \ldots \ldots \ldots$

2.10 Case RK3d: $s=1 / 6, c_{1}=1 / 3, c_{2}=2 / 3 \ldots \ldots \ldots \ldots \ldots \ldots$

2.11 Case RK3e: $s=1 / 6.289945083, c_{1}=1 / 3, c_{2}=2 / 3 \ldots \ldots \ldots \ldots$

3 Linear Stability Analysis of $L$ and $N$ for Various Methods . . . . . 14

3.1 Linear Stability of RK Methods . . . . . . . . . . . . . . . . . . . . . . . . . . . . . .

3.2 RK Order Stars . . . . . . . . . . . . . . . . . . . 17

3.3 Analysis of Methods Presented . . . . . . . . . . . . . . . . 17

3.3.1 RK3a, Linear Part . . . . . . . . . . . . . . . . 17

3.3.2 RK3a, Nonlinear Part . . . . . . . . . . . . . . . . 20

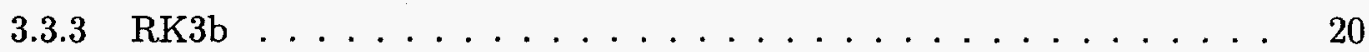

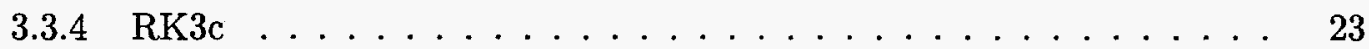

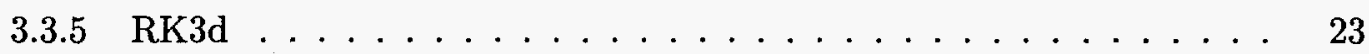

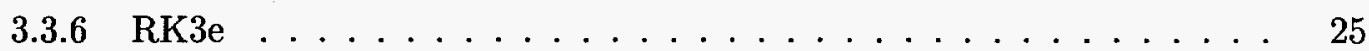

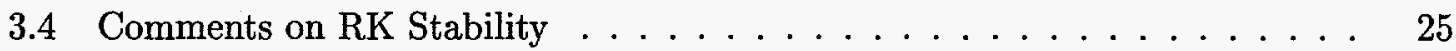

3.4.1 Implicitness Implies $r(z)$ Is Rational:

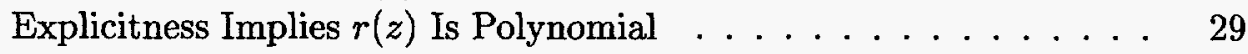

3.4.2 Relation Between $r(z)$ and $e^{z} \ldots \ldots \ldots \ldots 29$

3.4.3 Requirements on $s$ for Stiff Stability . . . . . . . . . . . 30

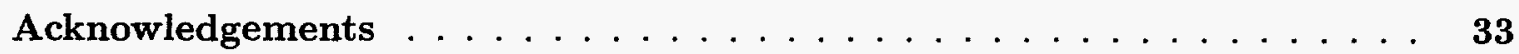


Appendix A Linear Multistep (LM) Methods . . . . . . . . . . . 35

A.1 Definition of LM Methods . . . . . . . . . . . . . . . . 35

A.2 Possible Advantages of LM Methods . . . . . . . . . . . . . . . . . . . . . . . . . . .

A.3 Derivation and Accuracy of LM Methods . . . . . . . . . . . . . . . . . . . . . . . . .

A.4 Linear Stability of LM Methods . . . . . . . . . . . . . . . . . . . . . . . . . . . . . . . . .

A.5 Examples of LM Methods and Their Numerical Analysis . . . . . . . . . . . 37

A.5.1 Milne-Simpson Method . . . . . . . . . . . . . . 38

A.5.2 Adams-Moulton Method . . . . . . . . . . . . . 38

Appendix B A Hunter's Guide to Further Investigations . . . . . . . . . . 41

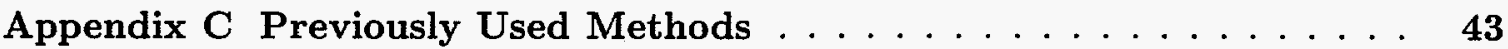

C.1 RK1a ............................... 43

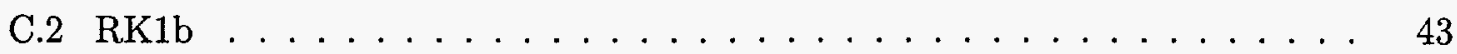

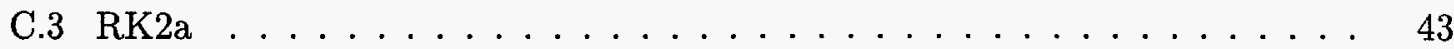

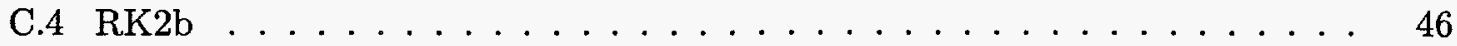

References ..........................48 


\section{List of Figures}

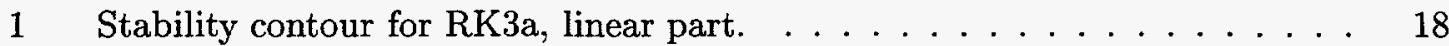

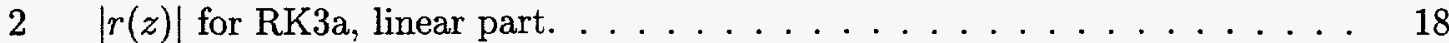

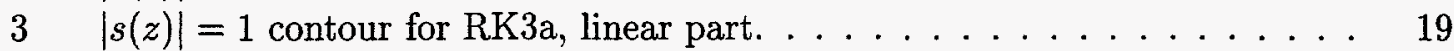

$4|s(z)|$ for RK3a, linear part. . . . . . . . . . . . . . . . 19

$5 \quad$ Large view of $|r(z)|$ for RK3a, linear part. . . . . . . . . . . . . 20

6 Stability contour for RK3a, nonlinear part. . . . . . . . . . . . . 21

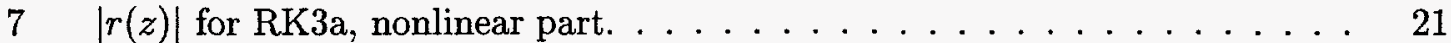

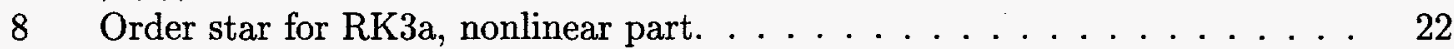

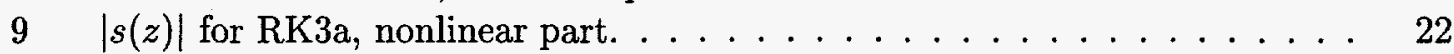

10 Stability contour for RK3c, linear part. . . . . . . . . . . . . 23

$11|r(z)|$ for RK3c, linear part. . . . . . . . . . . . . . . . 23

12 Order star for RK3c, linear part. . . . . . . . . . . . . . . . . 24

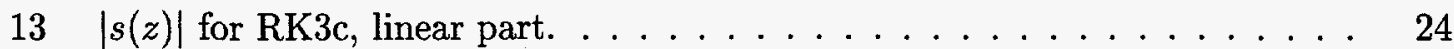

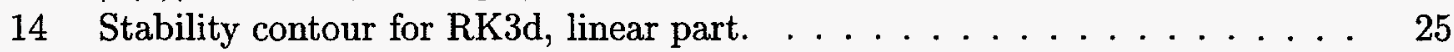

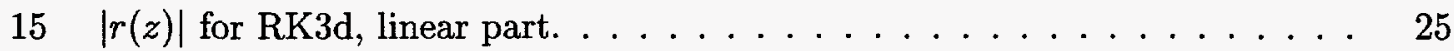

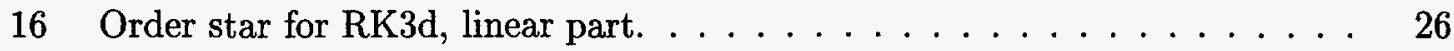

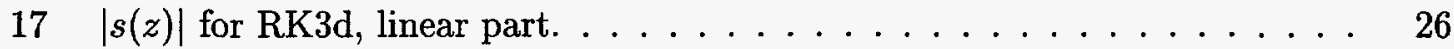

18 Stability contour for RK3e, linear part. . . . . . . . . . . . . 27

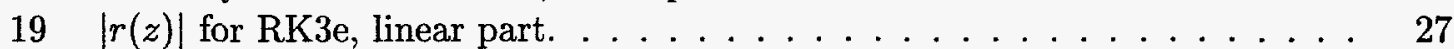

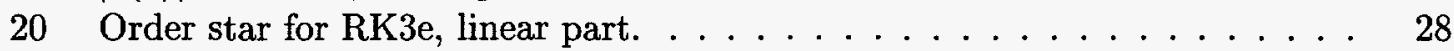

$21|s(z)|$ for RK3e, linear part. . . . . . . . . . . . . . . . 28

22 Stiff limit of $r$ as a function of implicitness parameter, s. . . . . . . . . 30

23 Stability contour and order star for RK1a, Euler method. . . . . . . . . . 44

24 Stability contour and order star for RK1b, Backward Euler Method. . . . . 45

25 Stability contour and order star for RK2a, Modified-Euler method. . . . . . 46

26 Stability contour and order star for RK2b, Heun predictor-corrector method. 47 


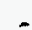




\begin{abstract}
There is an increasing need for more accurate numerical methods for large-scale nonlinear magneto-fluid turbulence calculations. These methods should not only increase the current state of the art in terms of accuracy, but should also continue to optimize other desired properties such as simplicity, minimized computation, minimized memory requirements, and robust stability. This includes the ability to stably solve stiff problems with long time-steps. This work discusses a general methodology for deriving higher-order numerical methods. It also discusses how the selection of various choices can affect the desired properties. The explicit discussion focuses on third-order Runge-Kutta methods, including general solutions and five examples. The study investigates the linear numerical analysis of these methods, including their accuracy, general stability, and stiff stability. Additional appendices discuss linear multistep methods, discuss directions for further work, and exhibit numerical analysis results for some other commonly used lower-order methods.
\end{abstract}




\section{Problem Formulation}

\subsection{General Motivation}

Many physical problems can be modelled as initial-value problems for a set of partial differential equations (PDEs). In many cases, these PDEs can be reduced to a set of coupled ordinary differential equations (ODEs). Without loss of generality, consider the system of ODEs to be first-order by introducing extra dependent variables. The resulting system of equations is often very large (or even countably or uncountably infinite). There are useful approximations that can lead to a closed, finite set of equations of manageable size to be solved. In general, the resulting system will be nonlinear and may not be amenable to analytic approaches. It is possible that a numerical solution could be employed to find approximate solutions.

The motivation for this work is to develop methods of solution for magneto-fluid problems typically addressed by the plasma turbulence group at Oak Ridge National Laboratory (ORNL) $[1,2,3,4]$. The starting point is a set of coupled PDEs describing the dynamic fluid behavior. Two of the spatial dimensions $(\theta$ and $\phi)$ are Fourier-expanded, and the other $(r)$ is approximated as a finite grid. This problem then reduces to a coupled set of ODEs. There is one ODE for the time evolution of each radial grid-point and $\theta-\phi$ mode. Obtaining fine spatial resolution requires that the number of ODEs be very large. The resulting ODEs have both a linear and nonlinear part. This can be represented as

$$
\frac{d \mathbf{y}}{d t}=\mathbf{L}[\mathbf{y}]+\mathbf{N}[\mathbf{y}],
$$

where $\mathbf{y}$ is a data vector of length $M$, the number of ODEs to be solved. The operators $\mathbf{L}$ and $\mathbf{N}$ map $\mathbf{M}$-vectors to $\mathbf{M}$-vectors. I assume $\mathbf{L}$ is a linear operator and $\mathbf{N}$ is a nonlinear operator.

Previous large-scale magneto-fluid simulations $[1,2,3,4]$ have used different numerical methods for the linear $(\mathbf{L})$ and nonlinear $(\mathbf{N})$ operators. The linear operator is usually of a block tridiagonal form that allows inversion. Therefore, $\mathbf{L}$ can be treated either explicitly or implicitly. However, $\mathbf{N}$ is nonlinear. An implicit solver applied to $\mathbf{N}$ would require a Jacobian linearization. This would involve an explicit convolution over poloidal modes. Although the resulting problem would be linear, and the matrix tridiagonal, the elements would each be unique. This means that each nonzero matrix element would need to be stored and explicitly inverted to apply an implicit algorithm. However, the problem sizes are so large that the required memory, even for a block tridiagonal matrix, are beyond the capacity of the largest present-day supercomputers. In that sense, these problems are limited both by processing power and memory per node, but the memory per node seems to be the most pressing restriction.

Previous work has explored using different numerical techniques for $\mathbf{L}$ and $\mathbf{N}$. I will review the methods used for these problems, using Young's classification terminology. [5] These techniques have differed in three respects: order of accuracy in $\Delta t$, implicitness, and time-centeredness. An early KITE code study [1] used two different techniques. The "mostly explicit" scheme was an explicit predictor-corrector algorithm that was secondorder-accurate in $\Delta t$ for both $\mathbf{L}$ and $\mathbf{N}$. The "mostly implicit" scheme time integrated $\mathbf{L}$ using the modified-Euler method and $\mathbf{N}$ using the Euler method. Therefore, it treated $\mathbf{L}$ in an implicit, time-centered, second-order-accurate fashion and treated $\mathbf{N}$ in an explicit, 
backward-biased, first-order-accurate fashion. An early FAR code study [2] was restricted to linear problems $(\mathbf{N}=0$ ) and treated $\mathbf{L}$ using the modified-Euler method (again secondorder-accurate, time-centered, and implicit). A subsequent study using FAR was extended to nonlinear problems [3]. It was reported that the nonlinear term was treated in an explicit fashion, but the details of the method, its time-centeredness, and the order of accuracy were not discussed. More recent extensions have treated $\mathbf{N}$ in a backward-biased, firstorder manner (Euler method) and a second-order, time-centered predictor-corrector manner. However, both were still explicit [6]. A recent study using the DTEM code [4] similarly treated $\mathbf{L}$ with the modified-Euler method (second-order-accurate, time-centered, implicit) and $\mathbf{N}$ with a predictor-corrector scheme (second-order-accurate, time-centered, explicit).

In this work, it is my purpose to extend these approaches to third-order accuracy. I use Runge-Kutta methods. I use explicit methods for $\mathbf{N}$ and implicit methods for $\mathbf{L}$ as was done in previous studies, since the problem of excess storage has not been addressed. Finally, since the methods are multi-stage, it is not strictly correct to speak in terms of treating $\mathbf{L}$ with one method and $\mathbf{N}$ with another. The intermediate values of $\mathbf{y}$ will depend on both $\mathbf{L}$ and $\mathbf{N}$. Therefore, the numerical behavior of $\mathbf{L}$ will be affected not only by the method used to evaluate $\mathbf{L}$ but also by the method used to evaluate $\mathbf{N}$ because of this intermediate mingling.

\section{$1.2 \quad$ Large Time-Steps}

Since this class of problem requires a large amount of computational resources, there is a premium on developing approaches that will reduce the computational effort required while maintaining accurate representation of dynamical features of interest. One such theme is the desire for longer time-steps. Fewer time-steps would be required to solve for a given simulation length. However, developing techniques to use longer time-steps is not a net benefit if the increase in computational cost to implement an algorithm is larger than the time saved.

Time-steps are usually limited by one of two factors. First, the time-step must be short enough to represent the phenomena of interest accurately. The deviation of the numerical solution from the actual solution for one step (called the "single step error," or SSE) is of the form $\epsilon=A(\Delta t)^{p+1}$, where $A$ is a constant characteristic of the method and the problem, and $p$ is a characteristic of the numerical method. The method is said to be an "order-p-accurate" method. I must choose $\Delta t$ small enough such that the accumulated SSE will not mask the physically relevant dynamical behavior.

Second, the time-step must be within the method's stability boundary. For each method applied to a certain problem, if $\Delta t$ is outside the stability boundary, then the SSE will not accumulate linearly but will exponentiate in a manner similar to a positive Liapunov exponent. The solution will become utterly nonphysical in only a few steps. For certain problems, there are classes of algorithms whose stability boundaries can extend to infinite $\Delta t$. Such methods are called stiffly stable.

One method to increase the time-step stability limit uses "implicit" differencing schemes for the numerical solution. "Implicit" means that new values of $\mathbf{y}$ are used in evaluating the operators on the right-hand side of Eq. (1). Solving for the updated values of $\mathbf{y}$ then requires an inversion of the $\mathbf{L}$ and $\mathbf{N}$ operators. Since $\mathbf{L}$ is a linear operator, it can be inverted using standard matrix algorithms. However, inversion of $\mathbf{N}$ is much more difficult 
because it is nonlinear. In practice, the cost of inverting $\mathbf{N}$ is much more than the time saved by having an implicit time-step. However, for special problems, there are techniques that allow efficient and rapid inversion of $\mathbf{L}$. For instance, in the magneto-fluid turbulence problem, $\mathbf{L}$ can be cast into a block tridiagonal form. In this work, I will search for a method that treats $\mathbf{L}$ implicitly and $\mathbf{N}$ explicitly for the reason discussed in Sect. 1.1. Furthermore, the method must be sufficiently implicit that the method will be stiffly stable for the large negative eigenvalues, the quickly decaying modes of $\mathrm{L}$.

One way to increase the time-step accuracy limit is to use higher-order differencing schemes. This work will extend previous second-order methods to third-order.

\subsection{Simplification to a Single ODE}

Developing numerical schemes is sometimes not straightforward. For purposes of deriving a numerical algorithm, it is easier to deal with a single ODE. Therefore, simplify consideration of Eq. (1) to a single ODE,

$$
\frac{d y}{d t}=\lambda y+N[y]
$$

where $N[y]$ is a nonlinear functional of $y$. It is usually straightforward to generalize a method applicable to a single ODE to a system of ODEs and maintain the same accuracy and stability properties.

If the problem is strictly linear $(\mathbf{N}=0)$, this can be done explicitly by executing a similarity transformation of $\mathbf{L}$ (over the field of complex numbers). In that case, $\mathbf{L}^{\prime}$ will be diagonal with (in general complex) eigenvalues along the main diagonal. Then the system of ODEs decouples into a large set of single, uncoupled ODEs. In that case, I can regard $\lambda$ in Eq. (2) as one of the eigenvalues. Therefore, the accuracy of the system of ODEs is dictated by the minimum accuracy of the method applied to single ODEs where the minimum is taken over all eigenvalues of $\mathrm{L}$. Likewise, the stability of the entire system is governed by the most unstable eigenvalue of $\mathbf{L}$.

For the general nonlinear case when $\mathbf{N} \neq 0$, this cannot always be accomplished. Nevertheless, I will make this simplifying assumption. It will be left for later analysis to validate that the accuracy and stability of the system of equations can be inferred from the accuracy and stability of the single ODE. For this reduced analysis, represent $\mathbf{L}, \mathbf{N}$, and $\mathbf{y}$ by $L, N$, and $y$, respectively to signify the reduction from a system of equations to a single equation.

\subsection{Requirements on Numerical Method}

I have therefore focused my concentration on developing methods to solve Eq. (2). Given $y_{n} \equiv y(n \Delta t)$ (and perhaps some historical values, $y_{n-1}, y_{n-2}, \ldots$ ) find an estimate of $y_{n+1}$. The desired characteristics of the derived numerical algorithm are as follows:

1. Third-order accuracy: $y_{n+1}^{\text {numerical }}-y_{n+1}^{\text {exact }} \propto(\Delta t)^{4}$.

2. Stiff stability with respect to $L$ by treating $L$ implicitly.

3. The use of the same implicitness parameter for each substep in the method in order that the matrix associated with $\mathbf{L}$ in the real problem will need to be inverted only once. 
4. The minimization of the number of evaluations of $N$ because in the full problem $\mathbf{N}$ involves convolutions of all modes and is very costly.

5. Either a self-starting algorithm (Runge-Kutta scheme) or an explicit specification for obtaining the starting values.

6. Maximum time-step stability (to the extent possible) of the instantaneous (linearized) spectrum of $N$, given that $N$ must be treated explicitly.

I also assume that

$$
L[y]=\lambda y,
$$

and that $N[y]$ is an unspecified nonlinear operator acting on y. Since I am dealing with a single ODE, $L$ must simply be a multiplicative constant. In terms of the usual time variation convention used in plasma physics,

$$
\lambda=\gamma-i \omega
$$

where $\gamma$ is the linear growth rate and $\omega$ is the linear oscillation frequency.

\section{Third-Order Runge-Kutta (RK) Methods}

\subsection{Description of RK Methods}

Runge-Kutta, or RK, methods numerically solve differential equations by evaluating the $L$ and $N$ operators and then using them to form a series of intermediate values for $y$ and finally combining the initial and intermediate values to calculate an estimate for the new value of $y$. Because they start with the current value and do not need any information about previous values, they are termed "self-starting" and are just as appropriate for the first-step as for the nth step. Deriving RK methods requires determining the coefficients used in developing the intermediate values and the final values for $y$ in order to match as many low-order Taylor series expansion terms as possible. Such methods are given in classic numerical analysis texts on this subject. $[5,7,8]$.

However, such approaches usually group the entire right-hand side of Eq. (2) as a single operator. In this work, I wish to treat $L$ implicitly and $N$ explicitly. Therefore, I will need two separate sets of coefficients, one for $L$ and one for $N$. The general RK method can then be expressed as

$$
\begin{gathered}
Y_{0} \equiv y_{n} \\
Y_{i} \equiv y_{n}+\Delta t\left(\sum_{j=0}^{s} a_{i j} L_{j}+\sum_{j=0}^{s} b_{i j} N_{j}\right) \\
y_{n+1}^{\text {numerical }} \equiv Y_{s}
\end{gathered}
$$


where $y_{n}$ is the numerical value at the old step and $y_{n+1}$ is the value at the new step. $Y_{i}$ is an intermediate calculation based on $y_{n}, L$, and $N . L_{j} \equiv L\left[Y_{j}\right]=\lambda Y_{j}$ and $N_{j} \equiv N\left[Y_{j}\right]$ is the notation for the operators evaluated at the intermediate calculation points. The parameter $s$ characterizes the method which is called an "s-stage RK method." The parameter $s$ determines the level of complexity used to calculate $y_{n+1}$. Here, I choose $s=3$. (Note: In general, the order of the method does not equal the number of stages in the method: $s \neq p$.) The coefficient matrices, $\mathbf{A}$ and $\mathbf{B}$, are $(s+1) \times(s+1)$ matrices with $\mathbf{A}_{i j}=a_{i j}$ and $\mathbf{B}_{i j}=b_{i j}$. (Note: Indices run from 0 to $s$.) The elements of these matrices define the RK method to be used. They specify how the previous value is used to find the intermediate calculation and how they are combined to ultimately form the value at the new step, $y_{n+1}$.

\subsection{The Structure of Coefficient Matrices}

The structure of the coefficient matrices gives some general information about the method. Nonzero matrix elements on the main diagonal and upper triangular portion indicate that the method is implicit. That is, $a_{i j} \neq 0$ for $j \geq i$ implies that a future approximation, $Y_{j}$, is required to solve for the current approximation, $Y_{i}$. Since I choose to treat $N$ explicitly, I require $b_{i j}=0$ when $j \geq i$. That is, $\mathbf{B}$ is strictly lower-triangular. I also require that the upper triangular part of $\mathbf{A}$ be zero $\left(a_{i j}=0\right.$ when $\left.j>i\right)$. If this were not the case, then I would have to solve simultaneously for the entire set of $Y_{i}$ 's, which would make the effective matrix solve $s^{2}$ as large. With these restrictions, the basic numerical scheme described in Eq. (4) reduces to

$$
Y_{i} \equiv y_{n}+\Delta t\left(\sum_{j=0}^{i} a_{i j} L_{j}+\sum_{j=0}^{i-1} b_{i j} N_{j}\right)
$$

\subsection{General Considerations of the Coefficient Equations}

The RK method is then specified when the entries in $\mathbf{A}$ and $\mathbf{B}$ are specified. There are $s(s+2)$ coefficients to be specified. [Note: Eq. (3) requires $a_{00}=0$.] For $s=3$ this means there are 15 coefficients to specify. These coefficients are specified by requiring that the $y_{n+1}^{\text {numerical }}$ correctly approximate the lower-order terms of the Taylor series expansion of $y_{n+1}^{\text {exact }}$,

$$
y_{n+1}^{\text {exact }}=y+\Delta t \frac{d y}{d t}+\frac{1}{2}(\Delta t)^{2} \frac{d^{2} y}{d t^{2}}+\frac{1}{6}(\Delta t)^{3} \frac{d^{3} y}{d t^{3}}+O(4)
$$

where the expansions take place at $t=n \Delta t$ and $y=y_{n}$. The derivatives of $y$ can be expressed in terms of $L$ and $N$ and their functional derivatives with respect to $y$ using Eq. (2). They become

$$
\begin{aligned}
y_{n+1}^{\text {exact }}= & y+\Delta t(L+N)+\frac{1}{2}(\Delta t)^{2}\left(L^{\prime}+N^{\prime}\right)(L+N)+ \\
& \frac{1}{6}(\Delta t)^{3}\left[N^{\prime \prime}(L+N)^{2}+\left(L^{\prime}+N^{\prime}\right)^{2}(L+N)\right]+O(4),
\end{aligned}
$$

where $N^{\prime} \equiv \frac{d N}{d y}$, etc., and $L^{\prime \prime}=0$ since $L$ is a linear operator. Now, I can make no assumptions about $L$ and $N$ (other than the fact that $L$ is linear). Therefore, I must choose the 
elements of $\mathbf{A}$ and $\mathbf{B}$ such that the coefficient of each type of term in Eq. (7) is equal to the coefficient of the same terms in a recursively Taylor expanded version of Eq. (5). If I do, then the method will be of order 3 . This imposes 16 conditions on the coefficients.

This becomes the starting point for a derivation of the general RK3 method. I must solve for the 15 coefficients subject to the 16 constraints. Note that while this is the statement of a simultaneous system of equations to be solved, the system is not linear but algebraic. Therefore, it is not clear beforehand whether a solution exists, or is unique. Moreover, I do not know beforehand how many and what types of parameters characterize the general method. Below I will see that it appears that the general case has five free parameters, of which some parameter choices are singular or lead to complex coefficients which may be objectionable. This is because the real number field is not closed under all inverse-algebraic operations (for instance, square roots).

\subsection{Explicit Construction of Coefficient Equations}

The next few subsections give an explicit and detailed derivation of the coefficient equations for the $s=3$ case. A general parameterized solution and five specific solutions for given parameters will be presented. These subsections are included for completeness, but if the reader is more interested in using the method than deriving the method, then the reader will want to skip to Sect. 2.7 .

The approach is to explicitly consider the calculation of $y_{n+1}^{\text {numerical }}$, Taylor expanding where necessary about $y_{n}$ and retaining all third and lower-order terms in $\Delta t$. Then, the numerical scheme is compared to the Taylor expansion of the exact scheme leading to the coefficient equations.

Before starting it will prove useful to define six new variables (with six definition equations):

$$
\begin{aligned}
c_{i} \equiv \sum_{j=0}^{i} a_{i j}, & i=1,2,3, \\
d_{i} \equiv \sum_{j=0}^{i-1} b_{i j}, & i=1,2,3 .
\end{aligned}
$$

The variables $c_{i}$ and $d_{i}$ will often appear as a unit in many of the equations. Also define

$$
\delta_{i} \equiv Y_{i}-y_{n}
$$

which expresses the difference of the ith incremental value from the old value. It will prove useful to decompose $\delta_{i}$ in orders of $\Delta t$. Define ${ }_{j} \delta_{i}$ as that portion of $\delta_{i}$ which is $\propto(\Delta t)^{j}$. Also note the notational convention of a left subscript as an order decomposition of a particular quantity.

Now analyze each step of Eq. (6).

$$
\begin{aligned}
Y_{1} & =y_{n}+\Delta t\left(a_{10} L_{0}+a_{11} L_{1}+b_{10} N_{0}\right) \\
& =y+\Delta t\left(a_{10} \lambda y+a_{11} \lambda Y_{1}+b_{10} N\right),
\end{aligned}
$$

where $y$ and $N$ are understood to mean $y_{n}$ and $N_{0}$, respectively. It is useful to define 


$$
z \equiv \lambda \Delta t=L^{\prime} \Delta t
$$

The expression for $Y_{1}$ then becomes

$$
Y_{1}=\left(1-a_{11} z\right)^{-1}\left[\left(1+a_{10} z\right) y+b_{10} N \Delta t\right]
$$

and

$$
\begin{gathered}
\delta_{1}=\left(1-a_{11} z\right)^{-1}\left(c_{1} z y+d_{1} N \Delta t\right)=\left(1-a_{11} z\right)^{-1} \Delta t\left(c_{1} L+d_{1} N\right) \\
{ }_{1} \delta_{1}=\Delta t\left(c_{1} L+d_{1} N\right) \\
{ }_{2} \delta_{1}=a_{11} z{ }_{1} \delta_{1} .
\end{gathered}
$$

In a similar fashion,

$$
Y_{2}=\left(1-a_{22} z\right)^{-1}\left\{\left[1+\left(c_{2}-a_{22}\right) z\right] y+d_{2} N \Delta t+\left(a_{21} z+b_{21} N^{\prime} \Delta t\right) \delta_{1}\right\}
$$

and

$$
\begin{gathered}
\delta_{2}=\left(1-a_{22} z\right)^{-1}\left[c_{2} z y+d_{2} N \Delta t+\left(a_{21} z+b_{21} N^{\prime} \Delta t\right){ }_{1} \delta_{1}\right] \\
{ }_{1} \delta_{2}=c_{2} z y+d_{2} N \Delta t \\
{ }_{2} \delta_{2}=a_{22} z{ }_{1} \delta_{2}+\left(a_{21} z+b_{21} N^{\prime} \Delta t\right)_{1} \delta_{1} .
\end{gathered}
$$

Finally,

$$
\begin{aligned}
Y_{3}= & \left(1-a_{33} z\right)^{-1}\left\{\left[1+\left(c_{3}-a_{33}\right) z\right] y+d_{3} N \Delta t+z\left(a_{31} \delta_{1}+a_{32} \delta_{2}\right)\right. \\
& \left.+N^{\prime} \Delta t\left(b_{31} \delta_{1}+b_{32} \delta_{2}\right)+\frac{1}{2} N^{\prime \prime} \Delta t\left(b_{31} \delta_{1}^{2}+b_{32} \delta_{2}^{2}\right)\right\}
\end{aligned}
$$

Now explicitly expand $Y_{3}$ in powers of $\Delta t$,

$$
Y_{3}={ }_{0} Y_{3}+{ }_{1} Y_{3}+{ }_{2} Y_{3}+{ }_{3} Y_{3},
$$

and require the terms to match the exact expansion

$$
y_{n+1}^{\text {exact }}=y+\Delta t y^{\prime}+\frac{1}{2}(\Delta t)^{2} y^{\prime \prime}+\frac{1}{6}(\Delta t)^{6} y^{\prime \prime \prime} .
$$

Equating these expressions to zero order yields

$$
{ }_{0} Y_{3}=y=y \text {. }
$$

The first-order relation gives

$$
{ }_{1} Y_{3}=\Delta t\left(c_{3} L+d_{3} N\right)=\Delta t y^{\prime} .
$$

This gives two equations:

$$
c_{3}=1
$$




$$
d_{3}=1
$$

For the second order,

$$
\begin{aligned}
{ }_{2} Y_{3}= & \left(a_{33} z\right){ }_{1} Y_{3}+\left[z\left(a_{31}{ }_{1} \delta_{1}+a_{32}{ }_{1} \delta_{2}\right)+N^{\prime} \Delta t\left(b_{31}{ }_{1} \delta_{1}+b_{32}{ }_{1} \delta_{2}\right)\right] \\
= & (\Delta t)^{2}\left[L^{\prime} a_{33}(L+N)+\left(a_{31} L^{\prime}+b_{31} N^{\prime}\right)\left(c_{1} L+d_{1} N\right)\right. \\
& \left.+\left(a_{32} L^{\prime}+b_{32} N^{\prime}\right)\left(c_{2} L+d_{2} N\right)\right] .
\end{aligned}
$$

There are four types of terms in this expansion, $-L^{\prime} L, L^{\prime} N, N^{\prime} L$, and $N^{\prime} N$ - which must all be set equal to $\frac{1}{2}$, since the second-order exact expansion term is

$$
\frac{1}{2}(\Delta t)^{2} y^{\prime \prime}=\frac{1}{2}(\Delta t)^{2}\left(L^{\prime}+N^{\prime}\right)(L+N)
$$

This leads to four coefficient equations:

$$
\begin{gathered}
a_{33}+a_{32} c_{2}+a_{31} c_{1}=1 / 2, \\
a_{33}+a_{32} d_{2}+a_{31} d_{1}=1 / 2, \\
b_{32} c_{2}+b_{31} c_{1}=1 / 2, \\
b_{32} d_{2}+b_{31} d_{1}=1 / 2 .
\end{gathered}
$$

The third-order relations become even more complex:

$$
\begin{aligned}
{ }_{3} Y_{3}= & \left(a_{33} z\right)_{2} Y_{3}+\left[\left(a_{31} z+b_{31} N^{\prime} \Delta t\right)_{2} \delta_{1}+\left(a_{32} z+b_{32} N^{\prime} \Delta t\right)_{2} \delta_{2}\right. \\
& \left.+\frac{1}{2} N^{\prime \prime} \Delta t\left(b_{31}{ }_{1} \delta_{1}^{2}+b_{32}{ }_{1} \delta_{2}^{2}\right)\right] \\
= & (\Delta t)^{3}\left\{\frac{1}{2} a_{33} L^{\prime}\left(L^{\prime}+N^{\prime}\right)(L+N)+a_{11} L^{\prime}\left(a_{31} L^{\prime}+b_{31} N^{\prime}\right)\left(c_{1} L+d_{1} N\right)\right. \\
& +a_{22} L^{\prime}\left(a_{32} L^{\prime}+b_{32} N^{\prime}\right)\left(c_{2} L+d_{2} N\right) \\
& +\left(a_{21} L^{\prime}+b_{21} N^{\prime}\right)\left(a_{32} L^{\prime}+b_{32} N^{\prime}\right)\left(c_{1} L+d_{1} N\right) \\
& \left.+\frac{1}{2} N^{\prime \prime}\left[b_{31}\left(c_{1} L+d_{1} N\right)^{2}+b_{32}\left(c_{2} L+d_{2} N\right)^{2}\right]\right\} .
\end{aligned}
$$

This is required to be equal to

$$
\frac{1}{6}(\Delta t)^{3} y^{\prime \prime \prime}=\frac{1}{6}(\Delta t)^{3}\left\{N^{\prime \prime}(L+N)^{2}+\left(L^{\prime}+N^{\prime}\right)^{2}(L+N)\right\} .
$$

This leads to nine constraints on the coefficients of the different types of terms:

$$
b_{32} c_{2}^{2}+b_{31} c_{1}^{2}=\frac{1}{3}
$$




$$
\begin{gathered}
b_{32} c_{2} d_{2}+b_{31} c_{1} d_{1}=\frac{1}{3} \\
b_{32} d_{2}^{2}+b_{31} d_{1}^{2}=\frac{1}{3} \\
b_{32} b_{21} c_{1}=\frac{1}{6}, \\
b_{32} b_{21} d_{1}=\frac{1}{6}, \\
\frac{1}{2} a_{33}+a_{11} b_{31} c_{1}+a_{22} b_{32} c_{2}+b_{21} a_{32} c_{1}+a_{21} b_{32} c_{1}=\frac{1}{3} \\
\frac{1}{2} a_{33}+a_{11} b_{31} d_{1}+a_{22} b_{32} d_{2}+b_{21} a_{32} d_{1}+a_{21} b_{32} d_{1}=\frac{1}{3}, \\
\frac{1}{2} a_{33}+a_{11} a_{31} c_{1}+a_{22} a_{32} c_{2}+a_{21} a_{32} c_{1}=\frac{1}{6}, \\
\frac{1}{2} a_{33}+a_{11} a_{31} d_{1}+a_{22} a_{32} d_{2}+a_{21} a_{32} d_{1}=\frac{1}{6},
\end{gathered}
$$

Counting the equations defining $c_{i}$ and $d_{i}$, this totals to 22 equations in 21 unknowns. However, Eq. (12) is trivially true. Note that Eq. (13) and Eq. (14) imply $d_{3}=c_{3}$, and Eq. (22) and Eq. (23) imply $d_{1}=c_{1}$. Eq. (19) and Eq. (20) together with the fact that $d_{1}=c_{1}$ gives $d_{2}=c_{2}$. Therefore,

$$
d_{i}=c_{i} \quad \text { for } \quad i=1,2,3 .
$$

This makes many of the equations redundant. The redundant pairs of equations are (13, $14),(15,16),(17,18),(19,20),(19,21),(22,23),(24,25)$, and $(26,27)$.

In total, I have eliminated 1 equation based on triviality and 8 equations based on redundancy. I have also eliminated 3 variables. This leaves a total of 13 equations for 18 variables. The variables are: $c_{1}, c_{2}, c_{3}, a_{33}, a_{32}, a_{31}, a_{30}, a_{22}, a_{21}, a_{20}, a_{11}, a_{10}, b_{32}, b_{31}, b_{30}$, $b_{21}, b_{20}$, and $b_{10}$. For clarity, it is useful to restate the 13 remaining equations:

$$
\begin{gathered}
c_{3}=1 \\
a_{30}=1-a_{33}-a_{32}-a_{31}, \\
a_{20}=c_{2}-a_{22}-a_{21}, \\
a_{10}=c_{1}-a_{11} \\
b_{30}=1-b_{32}-b_{31}
\end{gathered}
$$




$$
\begin{gathered}
b_{20}=c_{2}-b_{21}, \\
b_{10}=c_{1}, \\
a_{33}+a_{32} c_{2}+a_{31} c_{1}=\frac{1}{2}, \\
b_{32} c_{2}+b_{31} c_{1}=\frac{1}{2}, \\
b_{32} c_{2}^{2}+b_{31} c_{1}^{2}=\frac{1}{3}, \\
b_{32} b_{21} c_{1}=\frac{1}{6}, \\
\frac{1}{2} a_{33}+b_{32} a_{22} c_{2}+b_{31} a_{11} c_{1}+\left(b_{21} a_{32}+a_{32} b_{32}\right) c_{1}=\frac{1}{3},
\end{gathered}
$$

Equations (29)-(35) can be used to eliminate the 7 variables on the left-hand side by substitution, leaving a net of 6 equations in 11 variables. It would appear that I have a 5parameter family of possible RK methods. However, nothing in the problem has determined which 5 variables, or combinations of variables should be chosen as the parameters. It is important to remember that this system of equations is not linear. Therefore, even if the equations are independent in some sense, there is no general guarantee for the existence or uniqueness of solutions.

I choose to use $a_{33}, a_{22}, a_{11}, c_{1}$, and $c_{2}$ as parameters and use the remaining 6 equations to solve for the remaining 6 variables, $a_{32}, a_{31}, a_{21}, b_{32}, b_{31}$, and $b_{21}$. This choice of parameters is not based on an objective solvability criterion. It is heuristically motivated. This leads to some restrictions on parameter choices. For instance, even though $c_{1}$ is a parameter, it is not arbitrary. Choosing $c_{1}=0$ would make it impossible to satisfy Eq. (39). Similarly, unless $c_{1}=2 / 3, c_{1}=c_{2}$ is not allowed, as can be seen by examining Eq. (37) and Eq. (38). There are many such restrictions. Additionally, some parameter choices will cause some of the $a_{i j}$ 's or $b_{i j}$ 's to be complex. It is not at all clear what the complete set of restrictions on the choices for the values of the parameters will be.

Exploring such questions will lead to information about the entire range of RK3 methods for arbitrary parameterizations for this problem. Such an exploration is beyond the scope of this work. 


\subsection{General Parameterized Solution}

Before any more assumptions are made, one general feature can be observed. I can solve for the remaining unknown elements of $\mathbf{B}$ in terms of $c_{1}$ and $c_{2}$ using Eqs. (37-39). For this choice of parameters, the equations for $\mathbf{B}$ can be cast out as a separate subsystem to be solved. The result is

$$
\begin{aligned}
& b_{32}=\frac{-\frac{1}{2} c_{1}+\frac{1}{3}}{c_{2}\left(c_{2}-c_{1}\right)}, \\
& b_{31}=\frac{\frac{1}{2} c_{2}-\frac{1}{3}}{c_{1}\left(c_{2}-c_{1}\right)}, \\
& b_{21}=\frac{c_{2}\left(c_{2}-c_{1}\right)}{c_{1}\left(2-3 c_{1}\right)}
\end{aligned}
$$

However, there are certain special cases that must be considered separately. These cases are $c_{1}=c_{2}, c_{2}=0, c_{1}=0$, or $c_{1}=2 / 3$. These special cases will not be completely discussed in this work. However, it might be an interesting question to explore the numerical methods derived from these special cases. There is an intuition that they will either be pathologically inconsistent (such as the $c_{1}=0$ case) or naturally advantageous.

To review, I have solved for $\mathbf{B}$ given our five chosen parameters. I can now regard any $b_{i j}$ appearing in the remaining equations $(36,40$, and 41$)$ as additional parameters, since they are simply functions of the fundamental parameters. Solving Eq. (36) for $a_{31}$ gives

$$
a_{31}=\frac{1}{c_{1}}\left(\frac{1}{2}-a_{33}-a_{32} c_{2}\right) .
$$

Solving Eq. (40) for $a_{21}$ gives

$$
a_{21}=\frac{1}{c_{1} b_{32}}\left(\frac{1}{3}-\frac{1}{2} a_{33}-b_{32} a_{22} c_{2}-b_{31} a_{11} c_{1}-b_{21} a_{32} c_{1}\right) .
$$

Substituting these expressions into Eq. (41) and rearranging gives

$$
\begin{array}{r}
a_{32}^{2}\left(\frac{-b_{21} c_{1}}{b_{32}}\right)+a_{32}\left[\frac{1}{b_{32}}\left(\frac{1}{3}-\frac{1}{2} a_{33}-b_{31} a_{11} c_{1}-a_{11} b_{32} c_{2}\right)\right]+ \\
\left.\left[a_{11}\left(\frac{1}{2}-a_{33}\right)+\frac{1}{2} a_{33}-\frac{1}{6}\right)\right]=0
\end{array}
$$

Note that the coefficient of $a_{32}^{2}$ is $-1 /\left(6 b_{32}^{2}\right)$. This can be seen by examining Eq. (39). Likewise, the last two terms in the coefficient of $a_{32}$ reduce to $-a_{11} / 2$, as can be seen from Eq. (37). Define

$$
x \equiv a_{32} / b_{32}
$$

I am then left to solve a quadratic equation for $x$,

$$
x^{2}+x\left[-2+3\left(a_{11}+a_{33}\right)\right]+\left[1-3\left(a_{11}+a_{33}\right)+6 a_{11} a_{33}\right]=0 .
$$


Two features emerge. First, this equation does not depend on $c_{i}$ or $B$. So in some sense the solution for $A$ and $B$ has decoupled. Second, this equation no longer depends on $a_{22}$, meaning that it is still an arbitrary parameter. The explicit solution for $x$ is

$$
x=1-\frac{3}{2}\left(a_{11}+a_{33}\right)+\frac{3 \eta}{2}\left(a_{11}^{2}+a_{33}^{2}-\frac{2}{3} a_{11} a_{33}\right)^{1 / 2},
$$

where $\eta \equiv \pm 1$, depending on the root choice for the quadratic solution.

At this point, I have formally solved the simultaneous equations determining the coefficient matrices (except for the singular and pathological special cases mentioned above). However, this solution depends on five continuous parameters $-c_{1}, c_{2}, a_{33}, a_{22}$, and $a_{11}$ and a parity parameter, $\eta$. In what follows I will consider issues such as stiff stability and other numerical features. The algebra required to continue working with the general case is too cumbersome to be workable. Therefore, I will specialize the parameters.

\subsection{Equal Implicitness Parameterization}

Objective 3 requires

$$
a_{33}=a_{22}=a_{11} .
$$

That is, each implicit step involves the same coefficient multiplying the linear operator to be inverted. Call this parameter $s$. This should not be confused with the $s$ that is used to indicate the number of stages of a method, since $I$ have fixed that number at three for this part of the work. Use of an equal implicitness parameterization means that the linear matrix inversion of $\mathbf{L}$ will need to be calculated only once and can then be used for all intermediate steps. This is a considerable computational savings. This assumption does not affect the solution of the $\mathbf{B}$ subsystem, but it does reduce the $\mathbf{A}$ subsystem to

$$
\begin{gathered}
a_{31}=\frac{1}{c_{1}}\left(\frac{1}{2}-s-a_{32} c_{2}\right), \\
a_{21}=\frac{1}{c_{1} b_{32}}\left[\frac{1}{3}-\frac{s}{2}-s\left(b_{32} c_{2}+b_{31} c_{1}\right)-a_{32} b_{21} c_{1}\right], \\
x=1-3 s+\eta s \sqrt{3} .
\end{gathered}
$$

Note that the assumption of equal implicitness implies that there is no RK3 method with purely rational coefficients except for explicit calculations where $s=0$. This means that the elements of $\mathbf{A}$ will be irrational. Therefore, the coefficient matrices will be more complex than simple ratios of integers.

\subsection{Case RK3a: $s=1 / 2, c_{1}=1, c_{2}=1 / 2$}

To proceed further requires assuming values for the remaining parameters. In this subsection I assume $s=1 / 2, c_{1}=1$, and $c_{2}=1 / 2$. At this point, I have not found a motivating principle driving the choice of $c_{1}$ and $c_{2}$. These values are picked somewhat arbitrarily, although there have been other guesses for parameter choices that were not suitable either because of complexity or because of some numerical issue. Stability considerations motivate the choice of $s$ as described in Sect. 3.4.3.

For notational convenience, define 


$$
g \equiv \eta \sqrt{3}
$$

In that case the coefficient matrices become

$$
\begin{gathered}
\mathbf{A}=\left(\begin{array}{cccc}
0 & 0 & 0 & 0 \\
1 / 2 & 1 / 2 & 0 & 0 \\
(1+g) / 8 & (-1-g) / 8 & 1 / 2 & 0 \\
(4-g) / 6 & (1-g) / 6 & (-1+g) / 3 & 1 / 2
\end{array}\right) \\
\mathbf{B}=\left(\begin{array}{cccc}
0 & 0 & 0 & 0 \\
1 & 0 & 0 & 0 \\
1 / 4 & 1 / 4 & 0 & 0 \\
1 / 6 & 1 / 6 & 2 / 3 & 0
\end{array}\right)
\end{gathered}
$$

2.8 Case RK3b: $s=1 / 2, c_{1}=1 / 3, c_{2}=2 / 3$

Now choose $s=1 / 2, c_{1}=1 / 3$, and $c_{2}=2 / 3$. Then the coefficient matrices become

$$
\begin{gathered}
\mathbf{A}=\left(\begin{array}{cccc}
0 & 0 & 0 & 0 \\
-1 / 6 & 1 / 2 & 0 & 0 \\
(3+2 g) / 6 & (-1-g) / 3 & 1 / 2 & 0 \\
(1+3 g) / 8 & 3(1-g) / 4 & 3(-1+g) / 8 & 1 / 2
\end{array}\right) \\
\mathbf{B}=\left(\begin{array}{cccc}
0 & 0 & 0 & 0 \\
1 / 3 & 0 & 0 & 0 \\
0 & 2 / 3 & 0 & 0 \\
1 / 4 & 0 & 3 / 4 & 0
\end{array}\right) .
\end{gathered}
$$

2.9 Case RK3c: $s=1 / 3, c_{1}=1 / 3, c_{2}=2 / 3$

Now choose $s=1 / 3, c_{1}=1 / 3$, and $c_{2}=2 / 3$. Matrix $\mathrm{B}$ is the same as for RK3b and is given in Eq. (53). Then the linear coefficient matrix becomes

$$
\mathbf{A}=\left(\begin{array}{cccc}
0 & 0 & 0 & 0 \\
0 & 1 / 3 & 0 & 0 \\
(3+2 g) / 9 & -2 g / 9 & 1 / 3 & 0 \\
(2+3 g) / 12 & (1-g) / 2 & g / 4 & 1 / 3
\end{array}\right)
$$

2.10 Case RK3d: $s=1 / 6, c_{1}=1 / 3, c_{2}=2 / 3$

Now choose $s=1 / 6, c_{1}=1 / 3$, and $c_{2}=2 / 3$. Matrix $\mathbf{B}$ is the same as for RK3b and is given in Eq. (53). Then the linear coefficient matrix becomes

$$
\mathbf{A}=\left(\begin{array}{cccc}
0 & 0 & 0 & 0 \\
1 / 6 & 1 / 6 & 0 & 0 \\
(3+2 g) / 18 & (3-g) / 9 & 1 / 6 & 0 \\
(5+3 g) / 24 & (1-g) / 4 & (3+g) / 8 & 1 / 6
\end{array}\right)
$$




\subsection{Case RK3e: $s=1 / 6.289945083, c_{1}=1 / 3, c_{2}=2 / 3$}

Now choose $s=1 / 6.289945083, c_{1}=1 / 3$, and $c_{2}=2 / 3$. Matrix $\mathbf{B}$ is the same as for $\mathrm{RK} 3 \mathrm{~b}$ and is given in Eq. (53). This particular choice for $s$ is not arbitrary. Its value is determined by being slightly larger than an irrational zero of a particular polynomial that affects the stability properties of the method, as described below in Sect. 3.4.3. Then the linear coefficient matrix becomes
$\mathbf{A}=\left(\begin{array}{llll}0.0 & 0.0 & 0.0 & 0.0 \\ 0.174349433 & 0.1589839 & 0.0 & 0.0 \\ 0.342562694 & 0.165120071 & 0.1589839 & 0.0 \\ 0.416780169 & -0.174576438 & 0.598812369 & 0.1589839\end{array}\right)$

\section{Linear Stability Analysis of $L$ and $N$ for Various Methods}

The next consideration is the stability properties of this system. The general question of numerical stability is a very difficult issue and will not be addressed here. I will adopt two simplifying approaches that will allow useful, although not complete, statements. First, I restrict the discussion to linear stability analysis only. Second, I examine $L$ and $N$ operators separately.

These two assumptions can be a little confusing. Therefore, a few explanatory remarks are in order. First, the stability analysis examines the methods applied to $L$ and $N$ separately. That is, the analysis for the methods applied to $L$ strictly apply only when I am examining problems where $N=0$, exactly. Likewise, the analysis concerning the methods applied to $N$ applies only when $L=0$. When both $L$ and $N$ are nonzero, there exists the possibility of some sort of interaction that may give qualitatively different results. The numerical analysis of such general cases is beyond this work (although it may be the crucial focus for a given problem under study).

Second, I consider only linear numerical stability, even when considering the method used to treat $N$. That is, I do not draw conclusions about the nonlinear stability of the method applied to $N$ or the stability of the total method when the effects of $L$ and $N$ are mixed together using both operators simultaneously. Linear stability analysis for $L$ is certainly appropriate. It may not be clear at first glance that linear stability analysis applied to the method for solving $N$ provides useful information. However, near a given point of solution, say $y_{0}, N$ can be expanded in a Taylor series. Near $y_{0}, N$ acts almost linearly. That is it is only weakly nonlinear. Therefore, the linear stability analysis of the method used to treat $N$ will govern the numerical stability of the solution if I use the eigenvalues of the local linearization of $N$

In that sense, consider the archetypal equation

$$
\frac{d y}{d t}=\lambda y \text {. }
$$

When I are considering $L$, then $\lambda$ is understood to be one of the eigenvalues of $L$. When I am considering $N$, then $\lambda$ represents one of the eigenvalues of the instantaneous linearization of $N$. That is, at any point $y_{0}, N$ can be expanded as a functional series in 


$$
w=y-y_{0}
$$

as

$$
N[y]=N_{0}+\frac{\delta N}{\delta y} w+\frac{1}{2} \frac{\delta^{2} N}{\delta y^{2}} w^{2}+\ldots
$$

For small values of $w$, the equation $d y / d t=N[y]$ becomes

$$
\frac{d w}{d t}=\frac{\delta N}{\delta y} w+\frac{1}{2} \frac{\delta^{2} N}{\delta y^{2}} w^{2}+\ldots \approx \frac{\delta N}{\delta y} w .
$$

The linear term is dominant. Then $\lambda$ in Eq. (57) is interpreted an eigenvalue of $\delta N / \delta y$. The exception is when $y_{0}$ is an extremum or a saddle point of $N$. In that case careful analysis must be performed. However, usually a weak coupling limit is appropriate. Therefore, understanding the linear stability characteristics of the method that is applied to $N$ is important because it will give insight into the stability of the linearized problem, which is approximately equal to the problem to be solved.

For the archetypal linear problem, $d y / d t=\lambda y$, a general numerical scheme reduces to a recurrence relation among the $y_{n}$ 's:

$$
\sum_{i=0}^{k} \xi_{i} y_{n+1-i}=0 .
$$

This equation gives the scheme to determine $y_{n+1}$ given $y_{n}, y_{n-1}, \ldots, y_{n+1-k}$. The $\xi_{i}$ 's are coefficients that determine the numerical algorithm. In general, the $\xi_{i}$ 's depend on $z \equiv z \Delta t$, the normalized eigenvalue. The term $k$ is the number of steps in the method. For RK methods, $k=1$; all of the stages are intermediate values along the route to calculating the single step. Other methods, such as quadrature or methods based on differentiation or general linear multistep methods may have more than one step.

In order to analyze stability, define a polynomial,

$$
g(w) \equiv \sum_{i=0}^{k} \xi_{i} w^{k-i}
$$

The roots of $g(w)$ determine the stability characteristics of the algorithm. Note that the $\xi_{i}$ 's, and therefore the roots of $g(w)$, depend on $\lambda$. Define $z \equiv \lambda \Delta t$. A method is said to be stiffly stable if all of the roots of $g(w)$ have magnitude less than unity for $|z|$ arbitrarily large and $\operatorname{Re}(z)<0$.

\subsection{Linear Stability of RK Methods}

Stability of RK methods is defined in terms of a figure of merit,

$$
r(z) \equiv y_{n+1}^{\text {numerical }} / y_{n}^{\text {numerical }} \text {. }
$$

Since RK methods are single-step, $r$ is simply the single root of $g(r)=0$. Defined in this manner, $r$ is a measure of how the numerical solution grows or damps with time. If $|r|>1$, then the numerical solution will grow geometrically with time,

$$
y_{n}^{\text {numerical }}=(r)^{n} y_{0} .
$$


The case of $|r|>1$ is defined as unstable, while $|r|<1$ is considered stable. The case of $|r|=1$ is special and must be analyzed with care. Note that $r$ is a function of $z=\lambda \Delta t$. The set of points in the complex plane with $|r|<1$ determines which values of $z$ are suitable for numerical use. Since $\lambda$ is a characteristic of the ODE, then $\Delta t$ must be adjusted to ensure that the method is stable. In this manner, the stability contour sets a limit on the maximum allowable time-step for a faithful numerical solution.

Of particular interest is the behavior of $r$ for regions with $-\operatorname{Re}(z) \gg 1$. This is the case where there is a severely damped mode. If $r>1$ for large negative real values of $z$, then the time-step must be small to ensure that quickly decaying modes are not numerically unstable. This is an unfortunate situation because the most severely damped modes usually do not materially affect the solution. This type of problem is called a "stiff" problem because of the wide separation of scales. A method will be stiffly stable if $|r|<1$ for $\operatorname{Re}(z) \ll-1$. In that case, $\Delta t$ will not be restricted by rapidly decaying modes. Treating $L$ implicitly will help make the methods stifly stable. This is the crux of the second objective.

Because the analysis in this work is limited to linear stability of the operators considered in isolation, I will test the stability in terms of a coefficient matrix called $\mathbf{C}$. Let

$$
c_{i j} \equiv \begin{cases}a_{i j} & \text { for } N=0 \text { case } \\ b_{i j} & \text { for } L=0 \text { case }\end{cases}
$$

That is, if I am looking at the numerical algorithm applied to the $L$ operator, then $\mathbf{C}=\mathbf{A}$. If I am looking at the numerical algorithm applied to the $N$ operator then $\mathbf{C}=\mathbf{B}$.

Analysis of the general RK3 method gives an explicit expression for $r$ in terms of the elements of A or B. Such expressions can become tedious. So let us restrict the generality to the equal implicitness case of $c_{33}=c_{22}=c_{11} \equiv s$. By explicitly substituting for $Y_{1}$ and $Y_{2}$ in Eq. (6), I find

$$
r=\frac{1}{(1-s z)}\left\{\left(1+c_{30} z\right)+\frac{c_{31} z\left(1+c_{10} z\right)}{(1-s z)}+c_{32} z\left[\frac{1+c_{20} z}{(1-s z)}+\frac{c_{21} z\left(1+c_{10} z\right)}{(1-s z)^{2}}\right]\right\}
$$

For consistency, require that $r \rightarrow 1$ as $z \rightarrow 0$. The small $z$ expansion of Eq. (62) gives

$$
r=1+\left(c_{30}+c_{31}+c_{32}+s\right) z=1+z
$$

as required. Note Eq. (63) implies that the system is technically unstable for small $z$ when $\operatorname{Re}(z)>0$, but this conclusion is a bit misleading, since the exact solution also grows like $e^{z} \sim 1+z$. This issue will be discussed below with respect to order stars.

The big issue that puts the most limitations on a numerical method is the requirement of stiff stability. It can be analyzed by expanding Eq. (62) for large $z$ :

$$
r(z \rightarrow \infty)=\frac{-1}{s}\left[c_{30}-\frac{c_{31} c_{10}}{s}+c_{32}\left(\frac{-c_{20}}{s}+\frac{c_{21} c_{10}}{s^{2}}\right)\right]
$$

In order to be stiffly stable, choose $s$ and the $c_{i j}$ 's such that $|r(z \rightarrow \infty)| \leq 1$. 


\subsection{RK Order Stars}

A concept related to stability is that of "order stars" [7]. The linear analysis looks only at the size of the roots of $g(w)$. However, the exact answer also has a variation with $z$. That is,

$$
y_{n+1}^{\text {exact }} / y_{n}^{\text {exact }}=e^{\lambda \Delta t}=e^{z} .
$$

Therefore, it is sometimes useful to define

$$
C(z) \equiv r(z) e^{-z} .
$$

The term $C$ is called an "order star" for the method and $C$ is a measure of how faithfully the numerical solution reproduces the exact solution as a function of $z$ (at least for linear problems). The goal is to keep $C$ as close to 1 as possible.

The stability factor, $r$, and the order star, $C$, together give a good picture of the numerical method and provide guidance as to the allowable choices of time-step. For a given system, there is a spectrum of $\lambda$ 's. They are divided into two groups: "modes of interest" and "ignored modes." There are some modes on which I will focus. I want the time-step to be chosen to accurately follow the dynamics of those modes. There are other modes that are mathematically present in the exact and numerical solutions, but they have dynamics that keep their amplitudes small and in general are not the focus of interest. All I require of the numerical method with respect to these modes is that it must be stable. Accurate modelling of the "ignored modes" is not required. In terms of $r$ and $C$, this means that I require $z$ for modes of interest to be in regions of the complex plane where $C \approx 1$, and I require $z$ for ignored modes to be in regions where $r(z) \leq 1$. Since the spectrum of $\lambda$ is specified by the operator, I can only vary $\Delta t$ and the solution method. My goal is to pick a method that will maximize $\Delta t$ subject to these two requirements.

There is a distinct structure for the locations of the modes of interest and the ignored modes in the complex $z$-plane. The modes of interest will hover around the origin, or at least be located within the unit circle. The reason is accuracy. The SSE is proportional to $z^{p+1}$. If $|z| \simeq 1$ or larger, then the error in each step will swamp the behavior of the true solution. On the other hand, the ignored modes will lie in the left half-plane. This is because their real portion will have to be less than the modes of interest; otherwise, they will swamp the modes of interest (even for the exact solution). Thus, for most problems I am looking for a method with $C(z) \approx 1$ for $z$ in the unit circle and $|r(z)| \leq 1$ for $\operatorname{Re}(z)<0$. By implication, I am not overly concerned about the behavior of either $r(z)$ or $C(z)$ for values of $z$ with $\operatorname{Re}(z) \gg 1$.

\subsection{Analysis of Methods Presented}

Now let me go through the RK3 methods presented in Sect. 2.7 through Sect. 2.11 and explicitly calculate some figures of merit and plot some of them for both the linear and the nonlinear solution methods. For the nonlinear methods (the $b$-coefficients), I will assume that the operator is linear for stability analysis purposes.

\subsubsection{RK3a, Linear Part}

Here, let $c_{i j}=a_{i j}$, as detailed in Eq. (50). It can be shown that 


$$
r=\frac{1}{(1-z / 2)^{3}}\left(1-\frac{z}{2}-\frac{z^{2}}{4}+\frac{z^{3}}{24}\right) .
$$

Note that $r$ is independent of the choice for $\eta$ ! A contour plot of $|r(z)|=1$ is shown in Fig. 1. The curve represents the points where $|r(z)|=1$. In the left portion of the plane $|r(z)|<1$. Fig. 2 shows the actual values for $|r(z)|$. The plot actually shows $\min (|r(z)|, 1.0)$, so the plateau region is the unstable portion.

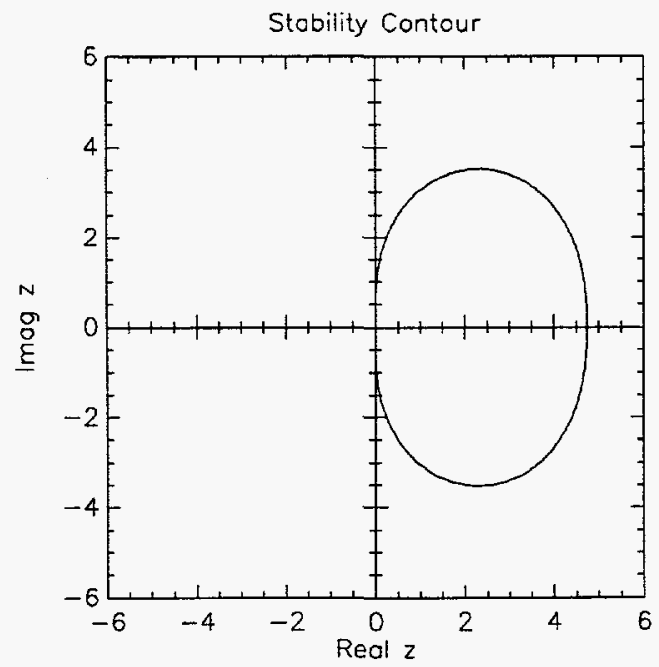

Figure 1: Stability contour for RK3a, linear part.

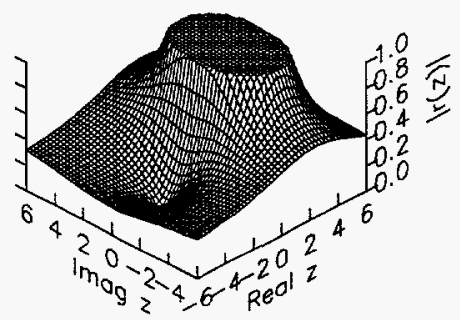

Figure 2: $|r(z)|$ for RK3a, linear part.

The order star is shown in Fig. 3 and 4. Fig. 1 shows that the method is stable for $|z|$ in the negative half-plane. Additionally, the order star is well behaved in the unit circle: $0.890<|C(z)|<1.071$ for $|z| \leq 1$.

However, there is a third-order pole in $C(z)$ located at $z=2$. This is a consequence of the three $(1-s z)^{-1}$ factors that appear in Eq. (62). This is a general feature of implicit methods. They will have a denominator that has a zero somewhere in the complex plane. An $s$-stage diagonally implicit method will have $s$ poles. If all of the implicitness parameters are the same, these poles will coalesce into a single $s$-order pole, as is the case here. The poles will be located at $z=(1 / s)=2$. 


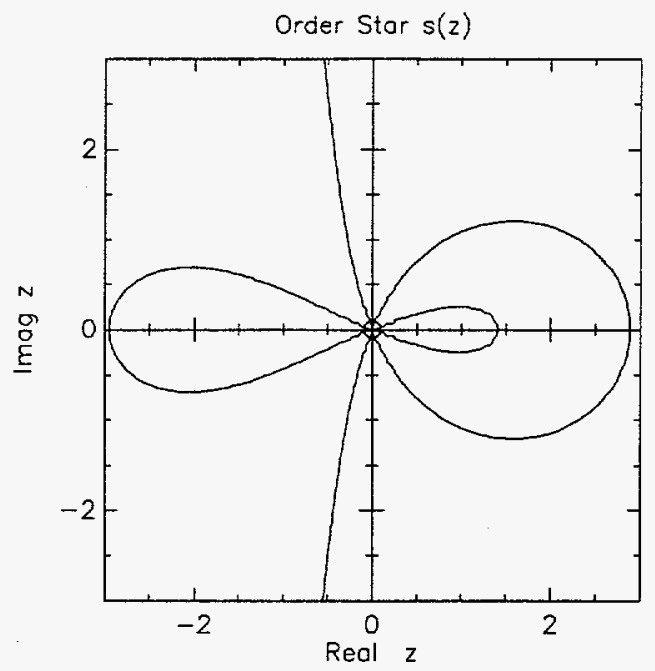

Figure 3: $|s(z)|=1$ contour for RK3a, linear part.

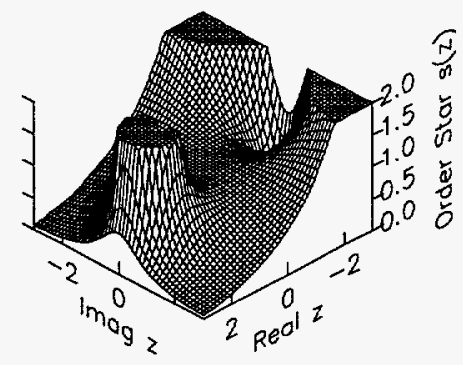

Figure 4: $|s(z)|$ for RK3a, linear part. 
The stiff stability can be seen by calculating the large $z$ limit:

$$
r(z \rightarrow \infty) \rightarrow-1 / 3
$$

Thus, the method is stiffly stable. This can also be seen in a larger-scale view of $|r(z)|$ in Fig. 5.

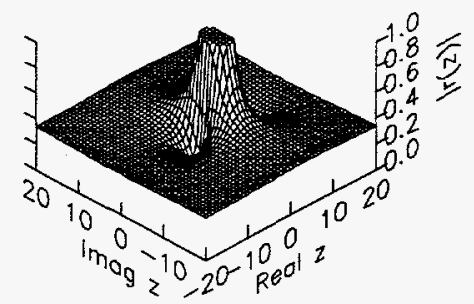

Figure 5: Large view of $|r(z)|$ for RK3a, linear part.

\subsubsection{RK3a, Nonlinear Part}

If I let $c_{i j}=b_{i j}$, as detailed in Eq. (51), I can examine the linear stability of the method that solves the nonlinear operator. It can be shown that

$$
r=1+z+\frac{1}{2} z^{2}+\frac{1}{6} z^{3}
$$

as would be expected for any explicit RK3 scheme. The stability contours, the values for $|r(z)|$, and the order stars are shown in Fig. 6-9. As can be seen, this scheme is not stiffly stable. I require $|z|<\sim 2$ for stability. However, the order star is still very accurate inside of the unit circle: $0.922<|C(z)|<1.071$ when $|z| \leq 1$. Therefore, I can conclude that the method is appropriate in terms of accuracy and normal stability criteria, but that it is inappropriate for stiff problems.

The implications are that RK3a will be useful if the linearized eigenvalues of $N$ are much smaller than the eigenvalues of $L$. If this is not the case, then there will not be much of a gain in time-step size by treating $L$ implicitly.

\subsubsection{RK3b}

Now let us examine method RK3b in the same fashion. For the linear part I again find

$$
r=\frac{1}{(1-z / 2)^{3}}\left(1-\frac{z}{2}-\frac{z^{2}}{4}+\frac{z^{3}}{24}\right) \text {. }
$$

For the nonlinear part,

$$
r=1+z+\frac{z^{2}}{2}+\frac{z^{3}}{6}
$$

This implies that the stability properties of RK3b are identical to the stability properties of RK3a in terms of $r(z)$ and $C(z)$. The only deviation will be from roundoff errors in the calculation procedure. This is an example of the general result that the stability of a diagonally implicit RK method with equal implicitness parameterization depends only on the choice of $s$ and not on $c_{1}$ and $c_{2}$, as shown in subsection 3.4.2 


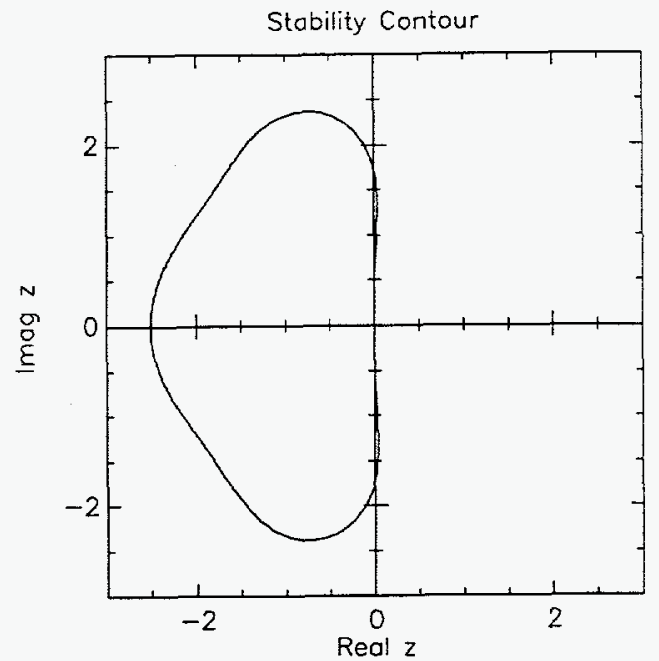

Figure 6: Stability contour for RK3a, nonlinear part.

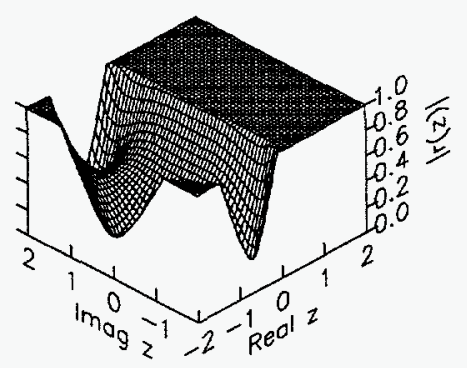

Figure 7: $|r(z)|$ for RK3a, nonlinear part. 


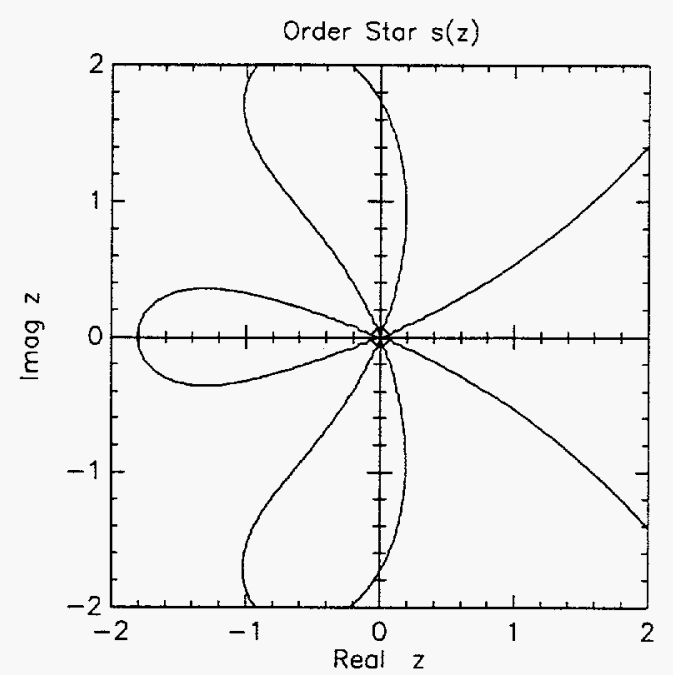

Figure 8: Order star for RK3a, nonlinear part.

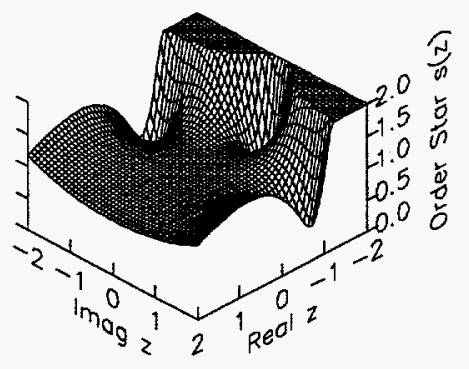

Figure 9: $|s(z)|$ for RK3a, nonlinear part. 


\subsubsection{RK3c}

RK3c will have different stability characteristics because it uses a different value for $s$. For RK3c,

$$
r=\frac{1}{(1-z / 3)^{3}}\left(1-\frac{z^{2}}{6}-\frac{z^{3}}{27}\right) .
$$

It is unity in the stiff limit: $r(|z| \rightarrow \infty)=1$. Inside of the unit circle; $0.9908<|C(z)|<$ 1.0078. Fig. $10-13$ show the stability limits and order stars.

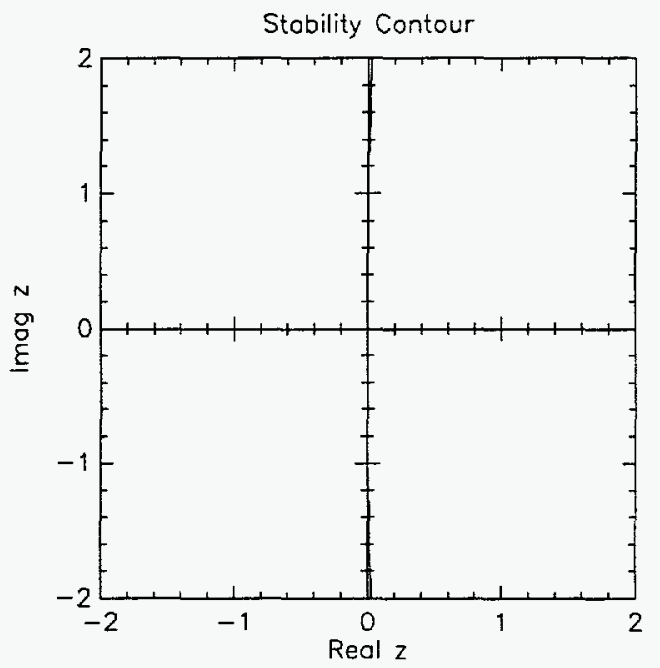

Figure 10: Stability contour for RK3c, linear part.

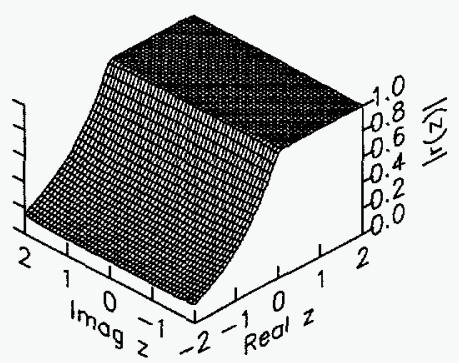

Figure 11: $|r(z)|$ for RK3c, linear part.

\subsubsection{RK3d}

For RK3d $r$ is

$$
r=\frac{1}{(1-z / 6)^{3}}\left(1+\frac{z}{2}+\frac{z^{2}}{12}-\frac{z^{3}}{216}\right)
$$




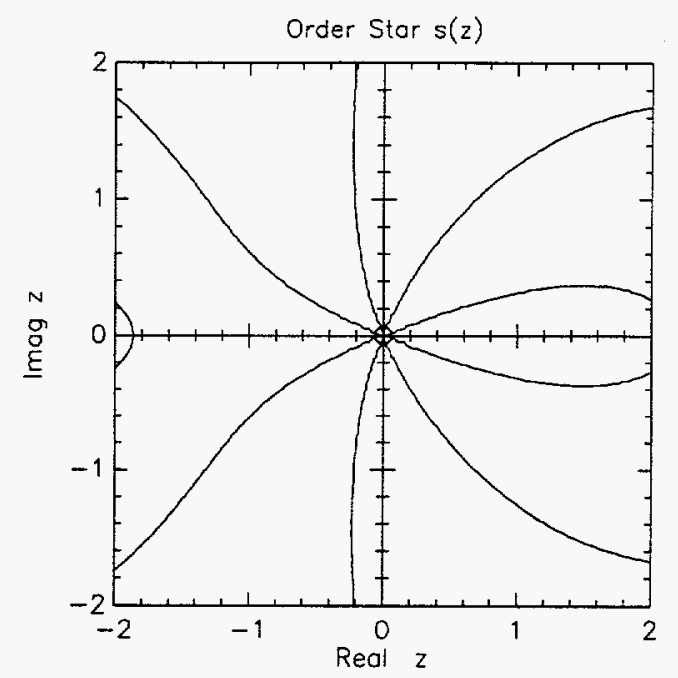

Figure 12: Order star for RK3c, linear part.

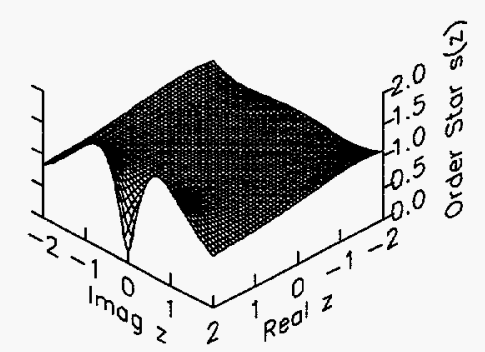

Figure 13: $|s(z)|$ for RK3c, linear part. 
It is unity in the stiff limit: $r(|z| \rightarrow \infty)=1$. Inside of the unit circle; $0.99503<|C(z)|<$ 1.0054. Fig. $14-17$ show the stability limits and order stars.

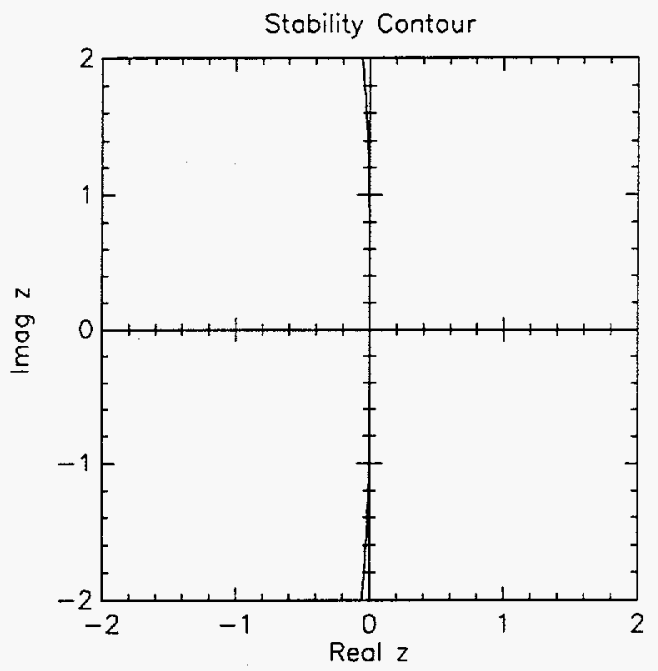

Figure 14: Stability contour for RK3d, linear part.

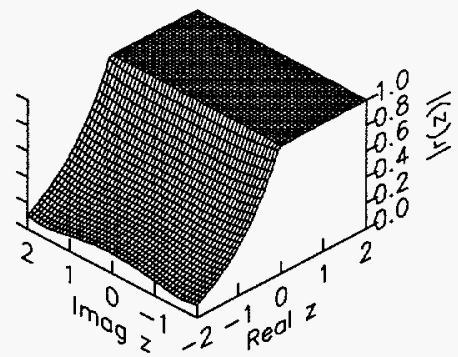

Figure 15: $|r(z)|$ for RK3d, linear part.

\subsubsection{RK3e}

For RK3e $r$ is

$$
r=\frac{1}{(1-z / 6.289945083)^{3}}\left(1+0.5230483000 z+0.09887594140 z^{2}\right) .
$$

It is less than $1.0 \times 10^{-8}$ in the stiff limit: $r(|z| \rightarrow \infty) \propto 0$. Inside of the unit circle; $0.99581<|s(z)|<1.0046$. Fig. $18-21$ show the stability limits and order stars.

\subsection{Comments on RK Stability}

Although I have examined only five methods, some very general features about the stability of these RK methods can be seen. These are discussed below. 


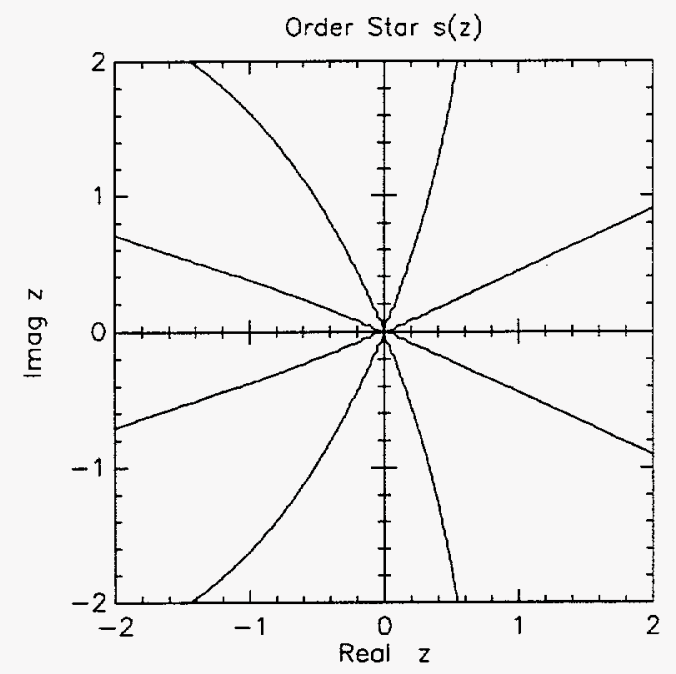

Figure 16: Order star for RK3d, linear part.

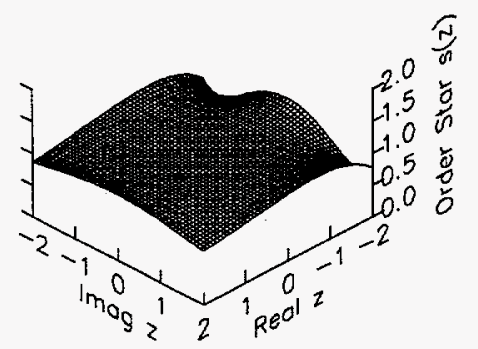

Figure 17: $|s(z)|$ for RK3d, linear part. 


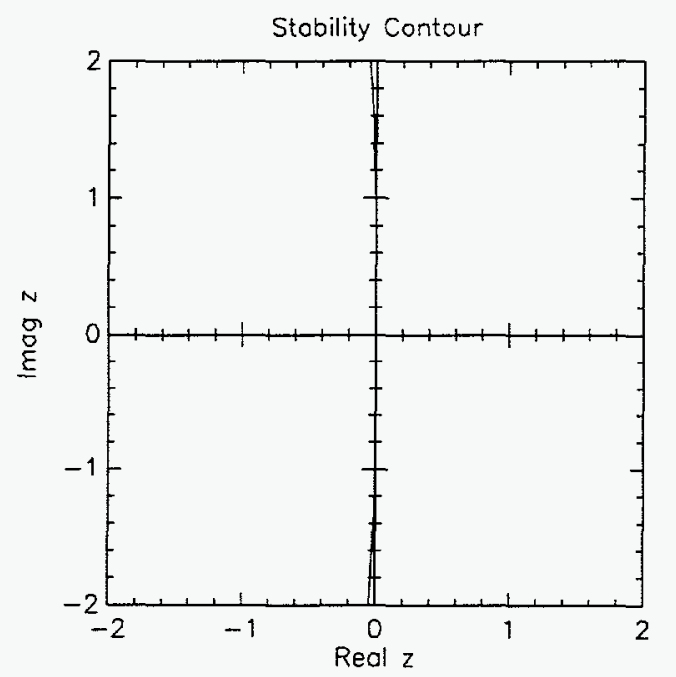

Figure 18: Stability contour for RK3e, linear part.

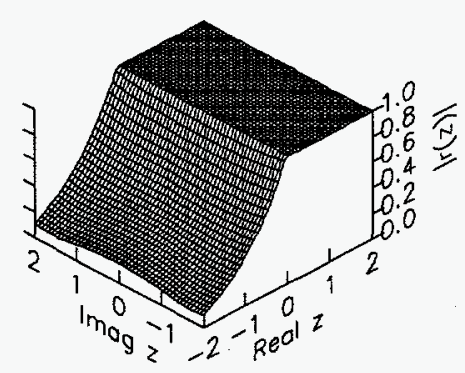

Figure 19: $|r(z)|$ for RK3e, linear part. 


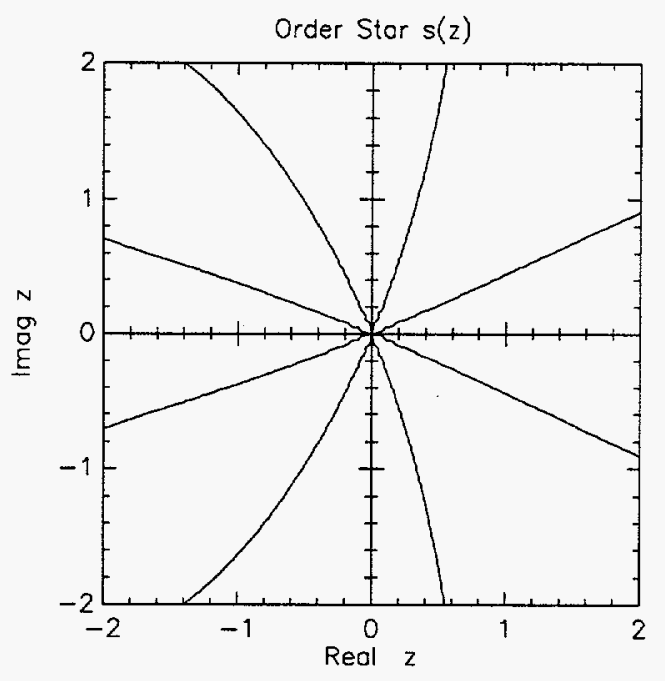

Figure 20: Order star for RK3e, linear part.

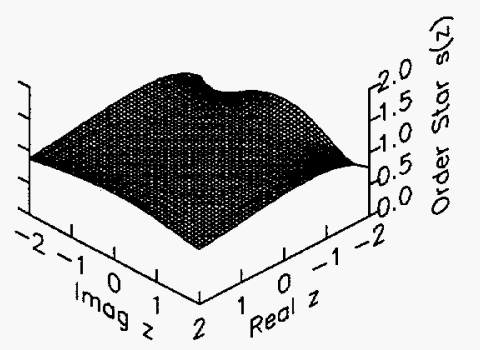

Figure 21: $|s(z)|$ for RK3e, linear part. 


\subsubsection{Implicitness Implies $r(z)$ Is Rational: Explicitness Implies $r(z)$ Is Polynomial}

In general, the stability parameter, $r(z)$, is a rational function. However, the denominator of $r(z)$ is $\xi_{0}$, the multiplier of $y_{n+1}$ in Eq. (59). It is apparent that if the method is explicit $\left(c_{i j}=0\right.$ when $\left.j \geq i\right)$, then $\xi_{0}$ is simply unity. Therefore, for explicit methods, $r(z)$ is necessarily a polynomial. Notice that all polynomials in $z$ of order one or greater are unbounded for $\operatorname{Re}(z) \ll-1$.

A generalization shows that this will hold true no matter what the order accuracy or the number of stages of the RK method. Therefore, I have proven the very general result that explicit methods cannot be stiffly stable in the sense of this discussion. The fact that implicit methods have a pole is the key to their success on stiff problems. As is known from functional analysis, an analytic function will have a singularity somewhere in the extended complex plane. For polynomials, this will be at infinity. However, this is precisely the region I wish to be bounded for stiff stability. Rational functions can be made to have bounded behavior for large arguments, but only at the expense of moving the pole or poles to some other position in the complex plane. Fortunately, for numerical problems the region of moderate $|z|$ with $\operatorname{Re}(z)>0$ is not of interest since the time-step must be chosen to keep all eigenvalues out of this region. This is precisely the region in which I can place the poles of the method such that they will not adversely affect the numerical solution. Therefore I must choose a method that has poles in the right half-plane. In terms of the recurrence relation, this means I must use an implicit method in order to get a denominator that is different from unity, and I must choose $s>0$ in order to place the poles in the right half-plane.

\subsubsection{Relation Between $r(z)$ and $e^{z}$}

In general, an $M$-order-accurate $\mathrm{RK}$ method must have the first $M$ terms of the Taylor expansion of $r(z)$ equal to the first $M$ terms of the expansion of $e^{z}$; otherwise, the SSE would not be $\propto z^{p+1}$ as required for a $p$-order-accurate method. If I let $q$ represent the number of stages in the RK method as discussed in Sect. 2.1, then for a $q$-stage diagonally implicit method, with equal implicitness parameterization, $r(z)$ will be of the form

$$
r(z)=\frac{P_{q}(z)}{(1-s z)^{q}}
$$

where $P_{q}(z)$ is a $q$ th-order polynomial in $z$ :

$$
P_{q}(z)=\sum_{k=0}^{q} \nu_{k} z^{k} .
$$

The values of $\nu_{k}$ are functions of $s$ and $q$. They must be adjusted such that $r(z)$ and $e^{z}$ have the same lower-order Taylor expansions.

For the three-stage, third-order methods presented here, the relationship between the $\nu_{k}$ 's, $s$, and the coefficients of the Taylor expansion of $e^{z}$ are

$$
\begin{gathered}
\nu_{0}=1, \\
\nu_{1}=1-3 s,
\end{gathered}
$$




$$
\begin{gathered}
\nu_{2}=\frac{1}{2}-3 s+3 s^{2}, \\
\nu_{3}=\frac{1}{6}-\frac{3}{2} s+3 s^{2}-s^{3} .
\end{gathered}
$$

It is interesting to note that stability depends only on $s$ and is independent of the other parameter, $c_{1}$ and $c_{2}$.

\subsubsection{Requirements on $s$ for Stiff Stability}

Examining $r(z)$ for large $|z|$ will determine whether a given method is stiffly stable. In that limit,

$$
r(|z| \rightarrow \infty) \rightarrow \frac{-\nu_{3}}{s^{3}}=1-\frac{3}{s}+\frac{3}{2 s^{2}}-\frac{1}{6 s^{3}} .
$$

I can now ask what values of $s$ will allow stiff stability by determining the locus of all $s$ such that $|r(|z| \rightarrow \infty)| \leq 1$. Fig. 22 plots $r(s,|z| \rightarrow \infty)$ as a function of $s$. For the set of values of $s$ of $\{-\infty, 1 / 6,1 / 3,+\infty\} r=1$. Somewhere in the range $(0.15,0.16) r=-1$. Therefore, I have two regions of $s$ that can be used for stiff problems: either $s>1 / 3$ or $s$ is slightly less than or equal to $1 / 6$.

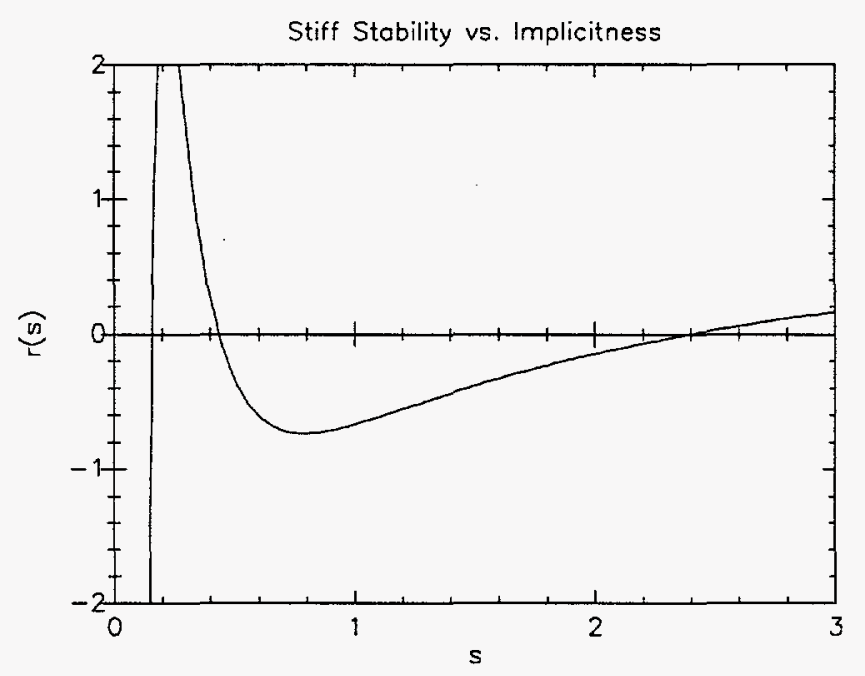

Figure 22: Stiff limit of $r$ as a function of implicitness parameter, $s$.

For $s=1 / 2, r$ approaches $-1 / 3$, as seen in RK3a and RK3b. For $s=1 / 6, r$ approaches 1 , as seen in RK3c. For $s=1 / 3, r$ approaches 1 , as seen in RK3d. For $s=1 / 6.289945083$, $r$ approaches 0 , as seen in RK3e. As is now apparent, the asymptotic behavior of $r(z ; s)$ formed the heuristic behind the choice of values for $s$ for RK3c, RK3d, and RK3e.

If I choose an $s$ such that $|r|=1$ in the stiff limit (RK3c and RK3d), it has an interesting property. The analytically most rapidly decaying modes are only moderately damped numerically. This may or may not be advantageous. On the other hand, if I choose $s$ such 
that $|r|=0$ in the stiff limit, the analytically decaying modes are severely damped. However, for the fluid turbulence problems, these different approaches may give insight into the calibration of numerical methods. Typically, a few unstable modes are nonlinearly coupled to stable modes such that there is an energy flow from mode to mode. That is, $N$ acts to grow damped modes and to damp growing modes. One nagging question has been to what extent the global simulation is reliable if the numerical scheme is overestimating or underestimating the damping rates of the decaying modes. By varying $s$ for an RK3 method, I can vary this amount of damping and in this manner test the sensitivity of the results to the accurate damping of decaying modes.

It is my conjecture that RK3e will be most suited for real calculations. It is very strongly stable in the stiff limit. Moreover, the pole in the right half-plane has been moved out beyond 6. This means that it perturbs $C$ less than methods with poles closer to the origin. The coefficient matrix in Eq. (56) was calculated numerically. This is because the exact algebraic value for $s$ such that $r(s)=0$ in the stiff limit is very complex. It is a solution of a root of a cubic equation. The expression for this root is a fraction that includes square roots and cube roots of complex numbers in both the numerator and denominator. One must be careful to include as much decimal precision as possible (preferably down to machine level) because any numerical errors will not only change the asymptotic value of $r$ but may also act to decrease the order of the method, since the numerical values will only satisfy the constraint equations on the coefficients to the precision of the coefficients themselves. Alternately, one could program the generalized parametric solutions for the coefficients directly and generate the coefficients on the fly from parameter input values. This would ensure that the coefficient constraint equations are satisfied to machine accuracy. 



\section{Acknowledgements}

I would like to acknowledge useful conversations with J. N. Leboeuf and L. A. Charlton. This research was sponsored by the U. S. Department of Energy, under contract DEAC05-84OR21400 with Martin Marietta Energy Systems, Inc., and supported in part by an appointment to the Oak Ridge National Laboratory Research Associate Program administered jointly by the Oak Ridge Institute for Science and Education and Oak Ridge National Laboratory. 


\section{Appendices}

\section{A Linear Multistep (LM) Methods}

\section{A.1 Definition of LM Methods}

There are, of course, many classes of numerical solvers, some of which might be profitably employed in extending the currently used methods. In this appendix, I briefly examine linear multistep methods. The idea behind linear multistep methods is to use the most recent value along with additional historical values to calculate the next value. Usually, the method to obtain the next value is a single calculation from the data on hand. This is in contrast to RK methods, where only the most recent data is used but with considerably more calculations used in each stage. That is, there are two types of extensions: In RK methods, stages are employed to increase the flexibility and ultimately the order of the method. In linear multistep methods, the number of historical values, or steps, is increased in order to get the same effect. It is possible to conceive of a generalized framework where there are many steps, each step possibly involving many stages. In such a generalization, RK methods are at one extreme, using only one step but many stages, and linear multistep methods are at the other extreme, using many steps each with only one stage. In some cases multi-step methods may look like RK methods if the intervening steps are viewed as intermediate values. Two examples are the Euler method and the midpoint method. In these (and other) cases, the same algorithm fits both paradigms. For the sake of brevity, I will not consider these generalized methods further in this work.

For the general (single) ODE,

$$
\frac{d y}{d t}=f(y, t)
$$

the general linear multistep procedure is defined as

$$
\sum_{i=0}^{N} \alpha_{i} y_{N+1-i}=\Delta t \sum_{i=0}^{N} \beta_{i} f_{N+1-i},
$$

where $f_{j} \equiv f\left(y_{j}, t_{j}\right)$. Eq. (70) defines the algorithm to determine $y_{N+1}$. I require $\alpha_{0} \neq 0$ and that either $\alpha_{N}$ or $\beta_{N}$ or both not be zero. Such a method is called an $N$-step method because each step of the algorithm involves values from $N$ steps of the solution. If $\beta_{0}=0$, then the method is said to be "open." This is synonymous with the method being "explicit" as discussed above. If $\beta_{0} \neq 0$, then the method is called "closed" and is an implicit method.

Without loss of generality, assume $\alpha_{0}=1$. This can be accomplished by a simple global renormalization. The number of coefficients to determine is $2 N$ for open methods and $2 N+1$ for closed methods.

\section{A.2 Possible Advantages of LM Methods}

There is a possibility that linear multistep methods may prove superior to RK methods because they may require fewer evaluations of the $L$ and $N$ operators. Each step involves many $y_{j}$ 's and $f_{j}$ 's, but most of those values have already been calculated by previous steps of the LM method. The total number of computations per step is less than for RK methods. 
If the time-step for both methods is the same, then the LM method will use less CPU time. However, there is a cost in that the LM methods must store back-values for many time-steps. If the problem size is large and storage is limited, this may prevent a successful application of LM methods. I will not attempt to predict the performance of LM methods except in the most general terms. It is an issue that is more appropriate for computer experimentation. It will vary depending on the computer architecture and the problem to be attacked.

\section{A.3 Derivation and Accuracy of LM Methods}

Unlike RK methods, the accuracy of LM methods can be examined using single Taylor expansions. That is, in RK methods, an intermediate value was determined and then used to determine a subsequent intermediate or final value. The analysis of such a method necessarily involved nested Taylor expansions, which grow very complex very quickly. However, since LM methods are only single-stage, there is no nesting of the expansions. An LM method will be order- $p$-accurate if it gives exact values for $y_{j}$ when applied to all polynomials of order $p$ or smaller. The conditions that this imposes on the coefficients of a $p$-order $N$-step method are given by Young [5] as

$$
\begin{gathered}
\sum_{i=0}^{N} \alpha_{i}=0, \\
\sum_{i=0}^{N}\left[(N-i) \alpha_{i}-\beta_{i}\right]=0, \\
\sum_{i=0}^{N}\left[(N-i)^{k} \alpha_{i}-k(N-i)^{k-1} \beta_{i}\right]=0, \text { for } 2 \leq k \leq p .
\end{gathered}
$$

This gives $p+1$ conditions for the $2 N$ coefficients of the open method or the $2 N+1$ coefficients of the closed method. There must exist an open $N$-step method of order $2 N-1$ and a closed $N$-step method of order $2 N$ since Eq. (71)-73 are linear in $\alpha_{i}$ and $\beta_{i}$ and are linearly independent. This defines the maximum order of an $N$-step method. However, it is also possible to define a method with free parameters or parameters determined according to some other prescription. The achievable order of the method will be reduced by the number of parameters that place conditions on the coefficients.

\section{A.4 Linear Stability of LM Methods}

I can analyze the linear stability of LM methods under the same rubric used for RK methods. If I assume that $f(y)=\lambda y$, then Eq. (70) can be represented in the form of Eq. (59) with

$$
\xi_{i}=\alpha_{i}-z \beta_{i}
$$

The stability of the method is then reduced to the consideration of the roots of $g(w)$ in Eq. (60). For an $N$-step method, $g(w)$ is an $N$ th-order polynomial. The coefficients of $g(w)$ are functions of $z$. Likewise, the roots of $g(w)$ are functions of $z$. It is sometimes useful to explicitly separate the $z$ dependence of $g(w)$ as 


$$
g(w)=\rho(w)-z \sigma(w) .
$$

By inspection, both $\rho$ and $\sigma$ are $N$ th-order polynomials of the form

$$
\begin{aligned}
& \rho(w)=\sum_{i=0}^{N} \alpha_{i} w^{N-i}, \\
& \sigma(w)=\sum_{i=0}^{N} \beta_{i} w^{N-i} .
\end{aligned}
$$

The complete stability analysis requires consideration of the entire set of roots of $g(w)$. However, this is often difficult, and if $N \geq 5$, it is not in general possible to find analytic forms for the roots. Useful limits can examined by use of $\rho$ and $\sigma$. It is to be expected that the roots will be continuous functions of $z$. Therefore, if the roots are determined for given values of $z$, this may lead to insight about the entire spectrum of roots for all $z$. There are two particular cases.

The first is the $z=0$ limit. This corresponds to infinitely small step size. In that case, $g(w)=\rho(w)$. If $\rho(w)$ has a root with magnitude greater than 1 , then the method is particularly pathological. It means that the numerical solution, although formally $p$ order accurate, will not converge to the true solution as $\Delta t \rightarrow 0$ because there is a spurious numerical root that is dominating the numerical solution. Such methods are called "strongly unstable" and should always be avoided. Unfortunately, the highest-order methods for a general $N$-step method are almost always strongly unstable, especially as $N$ gets larger. For example, the fifth-order open three-step method and the sixth-order closed three-step method are both strongly unstable.

The second limit is the $z$ large limit. In that case I have

$$
g(w)=z \sigma(w)
$$

Therefore, the roots of $g$ are exactly the roots of $\sigma$. The $z$ large limit is precisely the limit that must be examined to determine stiff stability.

Any candidate method should be examined for stiff stability and strong instability as a preliminary screen before expending the additional effort to solve the complete stability problem of finding all of the roots and exhibiting their behavior as a function of $z$.

\section{A.5 Examples of LM Methods and Their Numerical Analysis}

In this subsection I will look at two popular higher-order (greater than two) methods based on quadrature. Both fall short of achieving the objectives. In particular, they are not stiffly stable.

One additional twist that is often added is that of a predictor-corrector iteration. It allows one to predict a value for $y_{N+1}$ using a $N$-step open method and then use the predicted value to calculate $f_{N+1}$ for use in an $N$-step closed method. I will state both the predictor and corrector for algorithmic completeness, but I will only analyze the stability of the corrector. That is, I will look at the stability characteristics of the corrector, assuming a complete implicit solution instead of a weak implicit solution by means of a predictor method. 


\section{A.5.1 Milne-Simpson Method}

The Milne-Simpson method is a fourth-order, three-step predictor-corrector method. The predictor is

$$
y_{n+1}=y_{n-3}+\frac{\Delta t}{3}\left(8 f_{n}-4 f_{n-1}+8 f_{n-2}\right) \text {. }
$$

The corrector is

$$
y_{n+1}=y_{n-1}+\frac{\Delta t}{3}\left(f_{n+1}+4 f_{n}+f_{n-1}\right) .
$$

Evidently, for the corrector

$$
\begin{gathered}
\rho(w)=w^{3}-w \\
\sigma(w)=\frac{1}{3}\left(w^{3}+4 w^{2}+w\right) .
\end{gathered}
$$

The method is not strongly unstable. The roots of $\rho$ are $\{-1,0,1\}$. The +1 root is the "characteristic root" that is always present for a convergent method. It is the part of the numerical solution that follows the actual solution. While the -1 root does have unit magnitude, since it occurs singly, the method is conditionally stable. However, the method is not stiffly stable. The roots of $\sigma(w)$ are $\{-2-\sqrt{3}, 0,-2+\sqrt{3}\}$. The first root has magnitude greater than 1.

It might be instructive to utilize this method for a few test runs as a stalking horse to gauge the behavior of the operator in the problem under study. At this point I am not certain of the source of the current time-step limitation. This method might help delineate it. It should also be noted that this method is sometimes subject to an even-odd type of instability similar to that encountered with the leapfrog method. This is because the correcter has a factor of $w^{2}-1$ in $\rho$. This means that one root is at -1 and that a finite $z$ modification of this root may push it to instability for certain operators. Also, the MilneSimpson method has a "free" order of accuracy. That is, the coefficient of the first error term just happens to be zero so that the first nonzero error term is one order higher than expected. This means that $p$-order accuracy is obtainable with a method that is only as costly as a $p-1$-order-accurate method. Again, this is similar to the "free" order obtained with the leapfrog method.

\section{A.5.2 Adams-Moulton Method}

Another predictor-corrector method is the Adams-Moulton method. It is a four-step, fourth-order method. Its predictor is

$$
y_{n+1}=y_{n}+\frac{\Delta t}{24}\left(55 f_{n}-59 f_{n-1}+37 f_{n-2}-9 f_{n-3}\right),
$$

and the corrector is

$$
y_{n+1}=y_{n}+\frac{\Delta t}{24}\left(9 f_{n+1}+19 f_{n}-5 f_{n-1}+f_{n-2}\right) .
$$

For the corrector, 


$$
\begin{gathered}
\rho(w)=w^{4}-w^{3} \\
\sigma(w)=\frac{1}{24}\left(9 w^{4}+19 w^{3}-5 w^{2}+w\right) .
\end{gathered}
$$

The method is strongly stable. The roots of $\rho$ are the characteristic root of 1 and a triple root at 0 . However, again, the method is not stiffly stable as is shown by the fact that $\sigma(w)$ has a root between -2.3 and -2.4 . This method might prove useful as a stalking horse, since it is high-order, strongly stable (meaning well-behaved for small and moderate $z$ ), and pathological only for stiff problems. 



\section{B A Hunter's Guide to Further Investigations}

At this point I have not proceeded further in searching for additional LM methods or methods of other types. Clearly, such tasks could be undertaken and probably successfully completed. However, it seems wiser to first try some of the methods presented here, even some of those that do not meet the listed objectives, to get a feel for the sensitivity of the problem under study to the various strengths and weaknesses of the numerical methods.

At some future date, it may be desirable to again search for higher-order methods that do meet these objectives, or additional ones that may be imposed. There are several workable approaches:

First, one should determine what is desired. Some specific questions need to be answered. First, which is preferable, an RK method with many computations but less storage, or an LM method with more storage but less computation, or some hybrid method in between? Second, what is the relative size of the linearized spectrum of $N$ [as described in Eq. (58)] compared to the spectrum of $L$ ? If they are comparable, is it feasible to solve the entire system implicitly using a Newton-Raphson method for $N$ ? If not, how much is gained by solving for $L$ implicitly even if $N$ is solved for explicitly? Can an analytic expression for the Jacobian of $N$ be derived that will obviate the need to evaluate it numerically? Are predictor-corrector or other multistep/multi-stage methods appropriate?

Some of these questions can be addressed by looking at unsuitable methods. For instance, one can answer how long it takes to complete a three-stage RK step compared to a fourstep LM method, even if both methods are unstable, since this is simply a timing issue. Additionally, some unsuitable methods can provide real insight into the behavior of the problem. If a method that is not strictly stiffly stable is successful nonetheless, it says something about the stiffness of the problem at hand. If an RK3 method with $s=1 / 10$ is stable, that will also give some information.

Once these questions have been addressed, they will give additional guidance as to where to look when deriving new methods. I have explored the method of deriving and solving constraint equations in general, as well as looked at specific examples. This has been done in enough detail that it should be straightforward to extend this work to derive new methods. However, the algebra involved quickly becomes very tedious. I am of the opinion that RK4 and higher methods will require a symbolic algebra package. Similarly, obtaining third-order stiffly stable multistep methods will require that $N$ be significantly larger than it is in the Adams-Moulton or Milne-Simpson cases. Moreover, the constraints that all of the roots of $\sigma$ be on or within the unit circle imposes some fairly unnatural algebraic conditions of the coefficients. Again, the application of symbolic algebra and numerical root solvers may be required for systematic progress in that area. On the other hand, inspired heuristics might be used successfully to derive an appropriate method without the considerable effort required for a systematic treatment. 


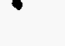

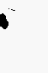




\section{Previously Used Methods}

In this appendix, I will comment on the stability and accuracy of some of the methods that have been applied to this problem previously. This material is included for completeness, so that those methods can be examined by the same criteria that I have used above. Curiously, all of these methods can be analyzed in terms of RK1 and RK2 methods, although their incarnations may look explicitly like predictor-corrector or low- $N$ multistep methods. The naming convention I use is $\mathrm{RKnl}$ where the numbers indicate the order of the method and the letters are character-labeling designations to separate different methods of the same order. One method is used for $L$ and another (or perhaps the same) method is used for $N$. I present the coefficient matrix and then discuss the linear stability in terms of $r, s$, and stiff stability for these methods.

\section{C.1 RK1a}

This is the classic Euler Method. It is an explicit one-stage method with first-order accuracy:

$$
\mathbf{C}=\left(\begin{array}{ll}
0 & 0 \\
1 & 0
\end{array}\right) .
$$

Fig. 23 shows $r(z)$ and $C(z)$. The stability characteristic is

$$
r(z)=1+z \text {. }
$$

The method is only stable about a circle of unit radius centered at $z=-1$. The order star is also less well behaved because the order is low: $0.104<C(z)<1.599$ when $|z| \leq 1$.

\section{C.2 RK1b}

This is the Backward-Euler method. It is an implicit one-stage method with first-order accuracy:

$$
\mathbf{C}=\left(\begin{array}{ll}
0 & 0 \\
0 & 1
\end{array}\right) .
$$

Fig. 24 shows $r(z)$ and $C(z)$. The stability characteristic is

$$
r(z)=\frac{1}{1-z} .
$$

The method is stable except for a circle of unit radius centered at $z=1$. The order star has a pole at $z=1$, just on the edge of the unit circle.

\section{C.3 RK2a}

This is the Modified-Euler method. It is an implicit one-stage method with second-order accuracy. It is time-centered

$$
\mathbf{C}=\left(\begin{array}{cc}
0 & 0 \\
1 / 2 & 1 / 2
\end{array}\right)
$$



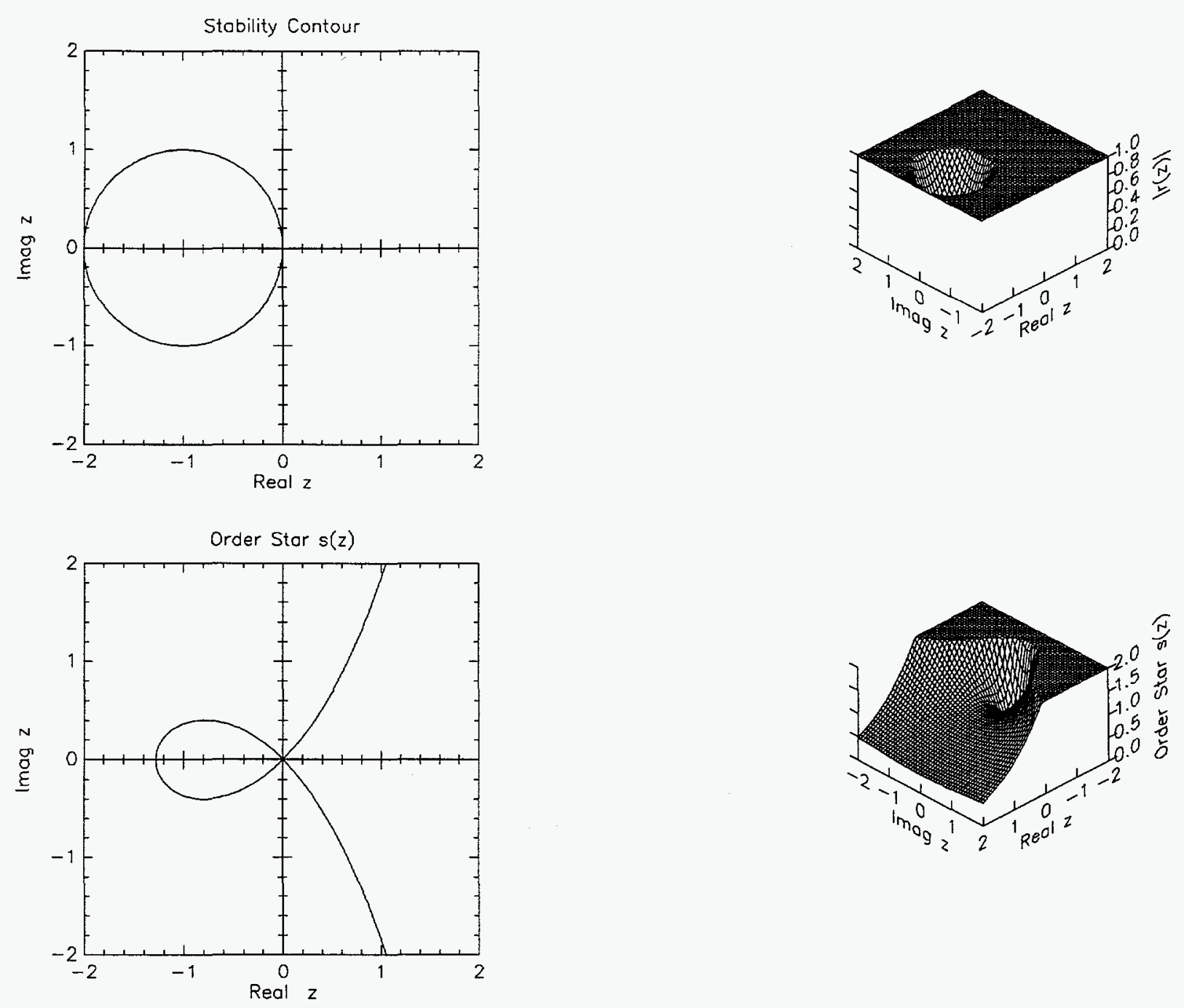

Figure 23: Stability contour and order star for RK1a, Euler method. 

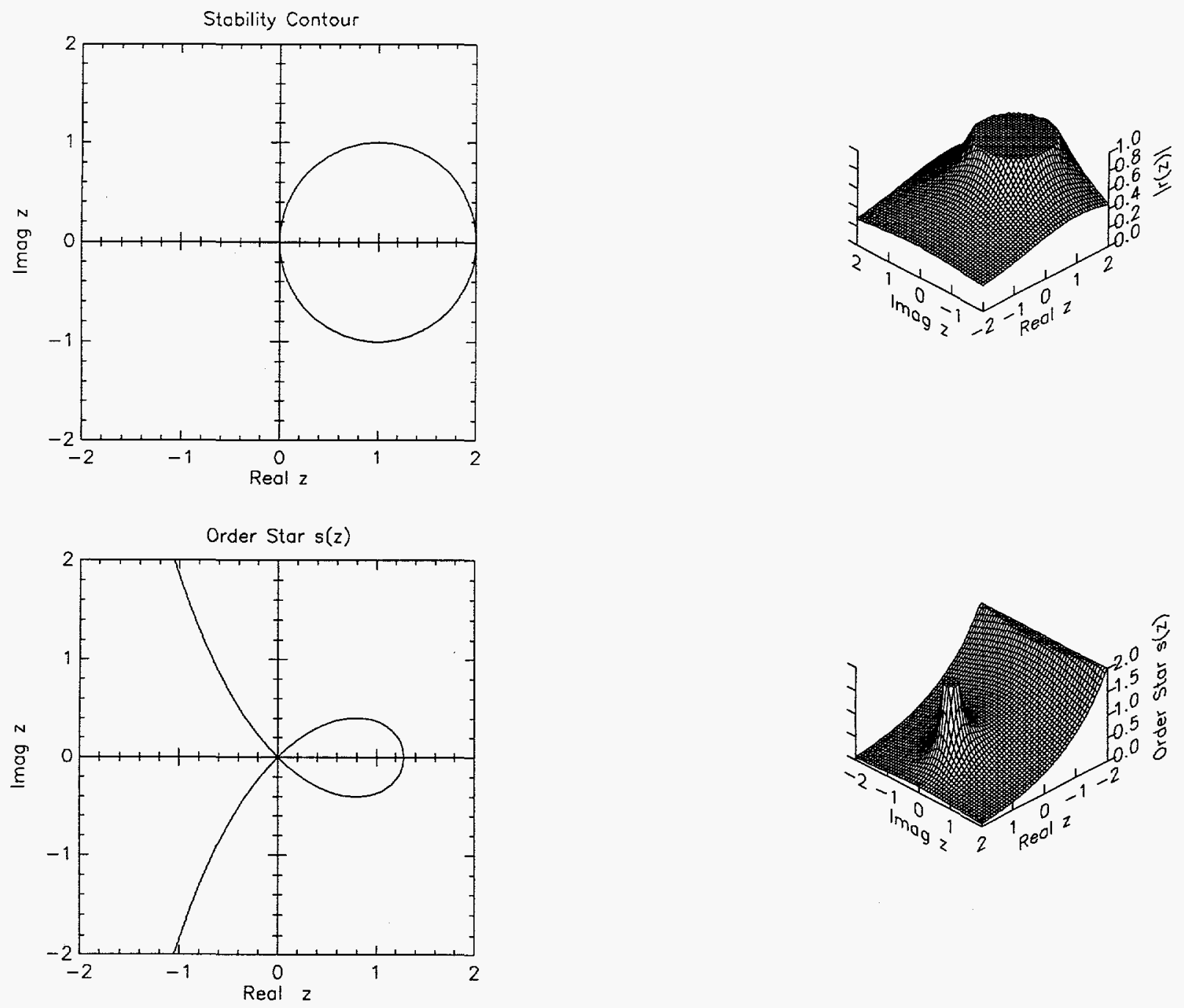

Figure 24: Stability contour and order star for RK1b, Backward Euler Method. 
Fig. 25 show $r(z)$ and $C(z)$. The stability characteristic is

$$
r(z)=\frac{1+z / 2}{1-z / 2}
$$

The method is stable for the entire left half-plane. The order star is fairly well behaved: $0.918<C<1.090$ for $|z| \leq 1$. Note that $|r| \rightarrow 1$ in the stiff limit. This is similar to RK3c and RK3d. Therefore, in this regard, I should consider RK3c and RK3d as third-order cousins of the midpoint method.
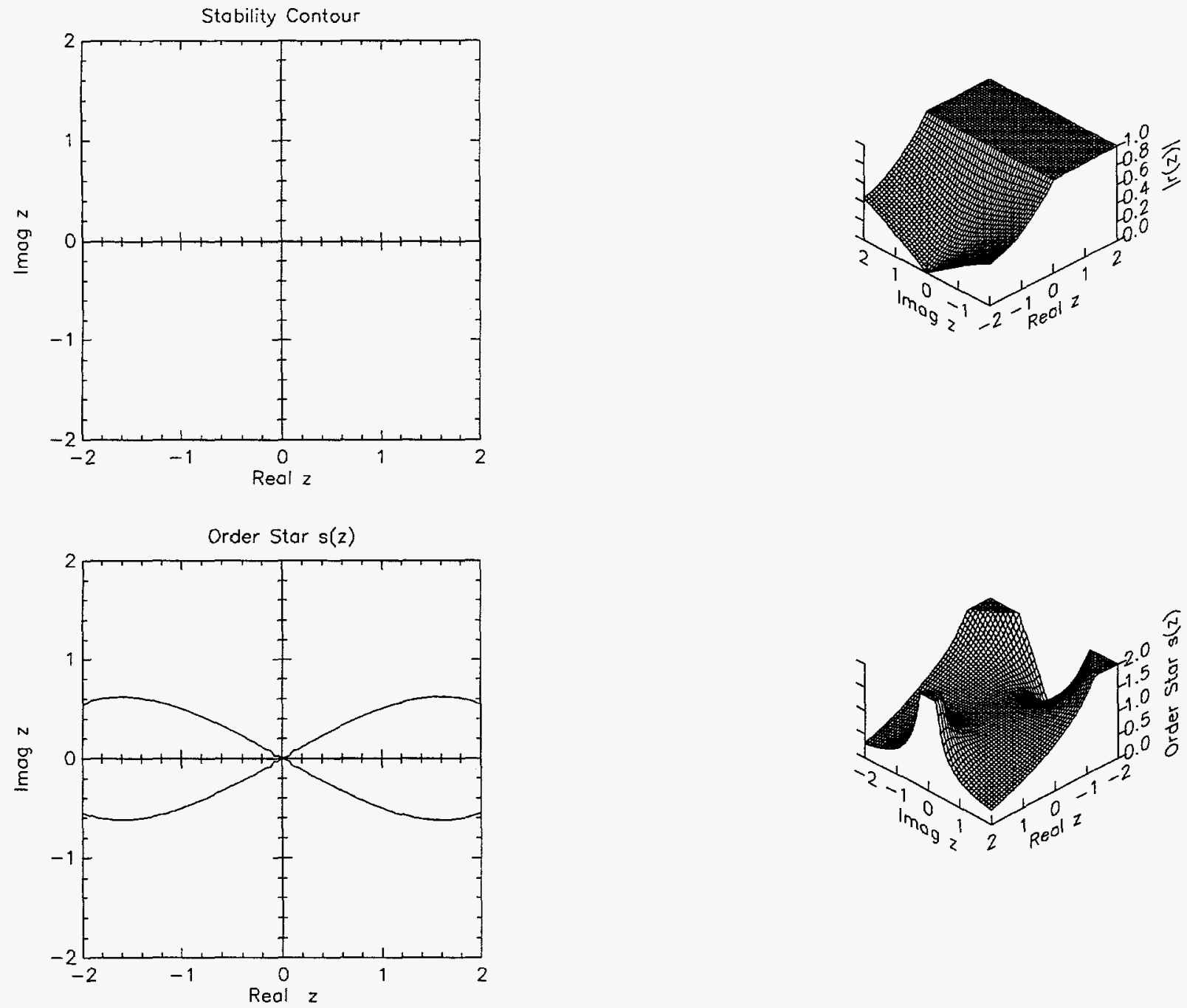

Figure 25: Stability contour and order star for RK2a, Modified-Euler method.

\section{C.4 RK2b}

This is the Heun Predictor-Corrector method. It is an explicit two-stage method with second-order accuracy. It is time-centered 


$$
\mathbf{C}=\left(\begin{array}{ccc}
0 & 0 & 0 \\
1 & 0 & 0 \\
1 / 2 & 1 / 2 & 0
\end{array}\right)
$$

Fig. 26 shows $r(z)$ and $C(z)$. The stability characteristic is

$$
r(z)=1+z+\frac{1}{2} z^{2}
$$

The method is stable for an oval shape with size on the order of 2 in the left half-plane. The order star is less well behaved: $0.760<C<1.308$ for $|z| \leq 1$.
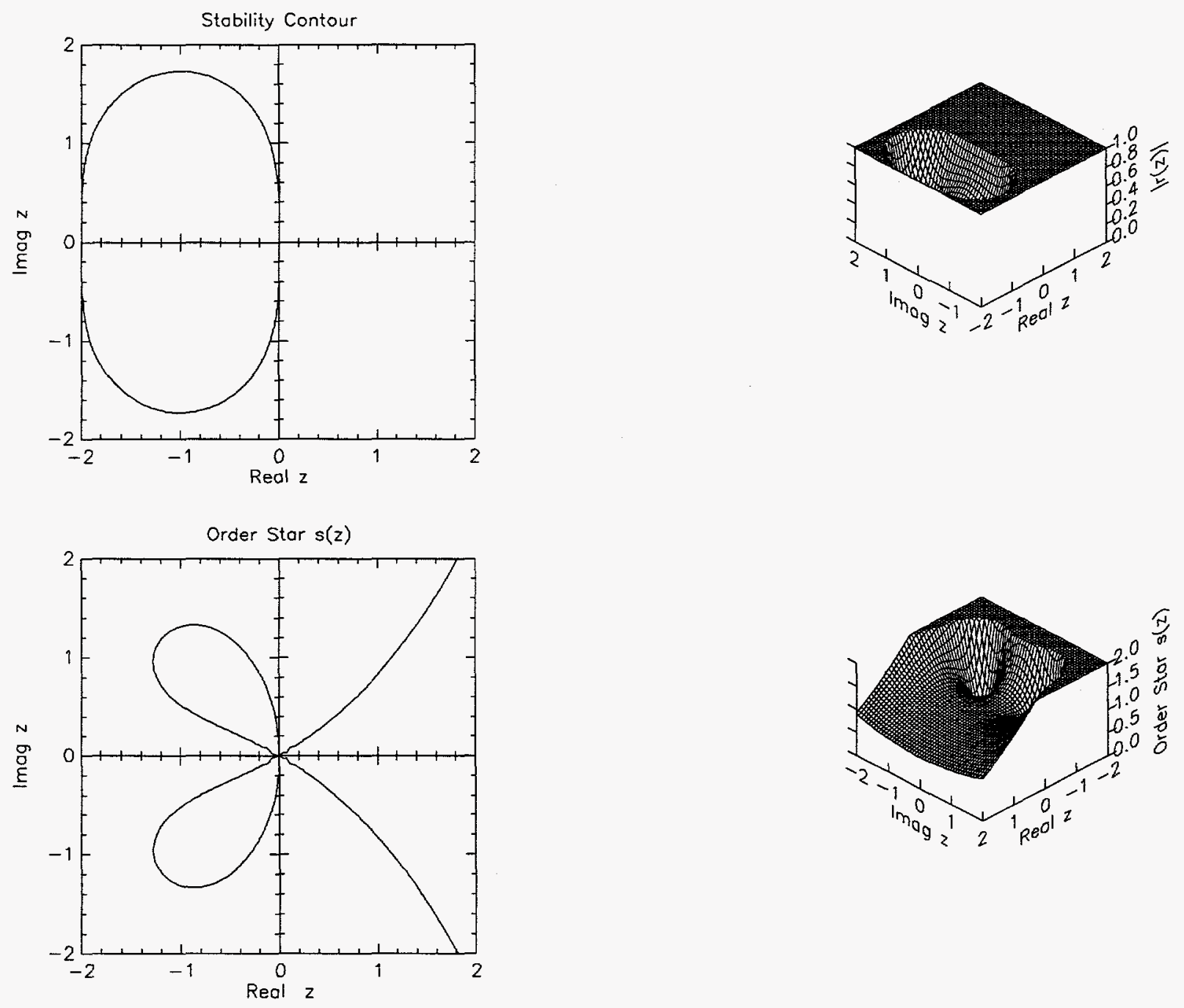

Figure 26: Stability contour and order star for RK2b, Heun predictor-corrector method. 


\section{REFERENCES}

[1] L. Garcia, H. R. Hicks, B. A. Carreras, L. A. Charlton, and J. A. Holmes, J. Comput. Phys. 65, 253 (1986).

[2] L. A. Charlton, J. A. Holmes, H. R. Hicks, V. E. Lynch, and B. A. Carreras, J. Comput. Phys. 63, 107 (1986).

[3] L. A. Charlton, J. A. Holmes, V. E. Lynch, B. A. Carreras, and T. C. Henden, J. Comput. Phys. 86, 270 (1990).

[4] K. L. Sidikman and B. A. Carreras, J. Comput. Phys. 114, 100 (1994).

[5] D. M. Young and R. T. Gregory, A Survey of Numerical Mathematics, Dover, 1972.

[6] L. A. Charlton, Private Communication.

[7] J. C. Butcher, The Numerical Analysis of Ordinary Differential Equations: Runge-Kutta and General Linear Methods, Wiley, 1992.

[8] R. A. Willoughby, Stiff Differential Systems, Plenum, 1973. 
ORNL/TM-12891

Dist. Category UC-427

\section{INTERNAL DISTRIBUTION}

1. Director, ORNL Fusion Energy Division

2. D. B. Batchelor

3. L. A. Berry

4. B. A. Carreras

5. H. E. Clark

6. R. J. Colchin

7. S. L. Milora

8-9. Laboratory Records Department

10. Laboratory Records, ORNL-RC

11-12. Central Research Library

13. Document Reference Section

14. Fusion Energy Division Library

15-16. ET/FE Division Publications Office

17. ORNL Patent Office

18. M. D. Carter

19. L. A. Charlton
20. K. R. Chen

21-51. J. W. Cobb

52. S. P. Hirshman

53. D. J. Hoffman

54. W. A. Houlberg

55. E. F. Jaeger

56. J. N. Leboeuf

57. V. E. Lynch

58. P. K. Mioduszewski

59. D. E. Newman

60. D. A. Spong

61. D. C. Stallings

62. C. Y. Wang

63. J. H. Whealton

64. J. C. Whitson

65. J. J. Yugo

\section{EXTERNAL DISTRIBUTION}

66. Office of the Assistant Manager for Energy Research and Development, Department of Energy, Oak Ridge Operations, P.O. Box 2000, Oak Ridge, TN 37831

67. N. A. Davies, Director, Office of Fusion Energy, Office of Energy Research, ER-50 Germantown, U.S. Department of Energy, Washington, DC 20545

68. M. Roberts, International Programs, Office of Fusion Energy, Office of Energy Research, ER-52 Germantown, U.S. Department of Energy, Washington, DC 20545

69. D. E. Baldwin, Lawrence Livermore National Laboratory, P.O. Box 5511, Livermore, CA 94550

70. R. W. Conn, Mechanical, Aerospace, and Nuclear Engineering Department, 6291 Boelter Hall, University of California, Los Angeles, CA 90024-1597

71. P. C. Liewer, MS 138-208, Jet Propulsion Laboratory, 4800 Oak Grove Drive, Pasadena, CA 91109

72. D. Sigmar, Plasma Fusion Center, Massachusetts Institute of Technology, 167 Albany St., NW16-288, Cambridge, MA 02139

73. K. I. Thomassen, L-637, Lawrence Livermore National Laboratory, P.O. Box 5511, Livermore, CA 94550

74. J. D. Callen, Department of Nuclear Engineering, University of Wisconsin, Madison, WI 53706-1687

75. S. O. Dean, Fusion Power Associates, Inc., 2 Professional Drive, Suite 248, Gaithersburg, MD 20879

76. H. K. Forsen, Bechtel Group, Inc., Research Engineering, P.O. Box 3965, San Francisco, CA 94119

77. R. W. Gould, Department of Applied Physics, California Institute of Technology, Pasadena, CA 91125 
78. R. A. Gross, Plasma Research Laboratory, Columbia University, New York, NY 10027

79. R. J. Hawryluk, Plasma Physics Laboratory, Princeton University, P.O. Box 451, Princeton, NJ 08543

80. D. M. Meade, Plasma Physics Laboratory, Princeton University, P.O. Box 451, Princeton, NJ 08543

81. W. M. Stacey, School of Nuclear Engineering and Health Physics, Georgia Institute of Technology, Atlanta, GA 30332

82. D. Steiner, Nuclear Engineering Department, NES Building, Tibbetts Avenue, Rensselaer Polytechnic Institute, Troy, NY 12181

83. R. Varma, Physical Research Laboratory, Navrangpura, Ahmedabad 380009, India

84. Bibliothek, Max-Planck Institut für Plasmaphysik, Boltzmannstrasse 2, D-8046 Garching, Federal Republic of Germany

85. Bibliothek, Institut für Plasmaphysik, KFA Jülich GmbH, Postfach 1913, D-5170 Jülich, Federal Republic of Germany

86. Bibliothek, KfK Karlsruhe GmbH, Postfach 3640, D-7500 Karlsruhe 1, Federal Republic of Germany

87. Bibliotheque, Centre de Recherches en Physique des Plasmas, Ecole Polytechnique Fédérale de Lausanne, 21 Avenue des Bains, CH-1007 Lausanne, Switzerland

88. R. Aymar, CEN/Cadarache, Departement de Recherches sur la Fusion Contrôlée, F-13108 Saint-Paul-lez-Durance Cedex, France

89. Bibliothèque, CEN/Cadarache, F-13108 Saint-Paul-lez-Durance Cedex, France

90. Library, JET Joint Undertaking, Abingdon, Oxfordshire OX14 3EA, England

91. Library, FOM-Instituut voor Plasmafysica, Rijnhuizen, Edisonbaan 14, 3439 MN Nieuwegein, The Netherlands

92. Library, National Institute for Fusion Science, Chikusa-ku, Nagoya 464-01, Japan

93. Library, International Centre for Theoretical Physics, P.O. Box 586, I-34100 Trieste, Italy

94. Library, Centro Richerche Energia Frascati, C.P. 65, I-00044 Frascati (Roma), Italy

95. Library, Plasma Physics Laboratory, Kyoto University, Gokasho, Uji, Kyoto 611, Japan

96. Plasma Research Laboratory, Australian National University, P.O. Box 4, Canberra, A.C.T. 2601, Australia

97. Library, Japan Atomic Energy Research Institute, Naka Fusion Research Establishment, 801-1 Mukoyama, Naka-machi, Naka-gun, Ibaraki-ken, Japan

98. G. A. Eliseev, I. V. Kurchatov Institute of Atomic Energy, P.O. Box 3402, 123182 Moscow, Russia

99. V. A. Glukhikh, Scientific-Research Institute of Electro-Physical Apparatus, 188631 St. Petersburg, Russia

100. I. Shpigel, Institute of General Physics, U.S.S.R. Academy of Sciences, Ulitsa Vavilova 38, Moscow, Russia

101. D. D. Ryutov, Institute of Nuclear Physics, Siberian Academy of Sciences, Sovetskaya St. 5, 630090 Novosibirsk, Russia

102. O. Pavlichenko, Kharkov Physical-Technical Institute, Academical St. 1, 310108 Kharkov, Ukraine

103. Deputy Director, Southwestern Institute of Physics, P.O. Box 15, Leshan, Sichuan, China (PRC)

104. Director, The Institute of Plasma Physics, P.O. Box 1126, Hefei, Anhui, China (PRC)

105. R. H. McKnight, Experimental Plasma Research Branch, Division of Development and Technology, Office of Fusion Energy, Office of Energy Research, ER-542 Germantown, U.S. Department of Energy, Washington, DC 20545

106. E. Oktay, Division of Confinement Systems, Office of Fusion Energy, Office of Energy Research, ER-55 Germantown, U.S. Department of Energy, Washington, DC 20545

107. W. Sadowski, Fusion Theory and Computer Services Branch, Division of Applied Plasma Physics, Office of Fusion Energy, Office of Energy Research, ER-541 Germantown, U.S. Department of Energy, Washington, DC 20545 
108. R. E. Mickens, Atlanta University, Department of Physics, Atlanta, GA 30314

109. M. N. Rosenbluth, University of California at San Diego, La Jolla, CA 92037

110. D. Schnack, SAIC, 10260 Campus Point Drive, San Diego, CA 92121

111. Duk-In Choi, Department of Physics, Korea Advanced Institute of Science and Technology, P.O. Box 150, Chong Ryang-Ri, Seoul, Korea

112. Library of Physics Department, University of Ioannina, Ioannina, Greece

113. C. De Palo, Library, Associazione EURATOM-ENEA sulla Fusione, CP 65, I-00044 Frascati (Roma), Italy

114. Laboratorio Associado de Plasma, Instituto Nacional de Pesquisas Espaciais, Caixa Postal 515, 122201, Sao Jose dos Campos, SP, Brazil

115. Theory Department Read File, c/o D. W. Ross, University of Texas, Institute for Fusion Studies, Austin, TX 78712

116. Theory Department Read File, c/o R. Parker, Director, Plasma Fusion Center, NW 16-202, Massachusetts Institute of Technology, Cambridge, MA 02139

117. Theory Department Read File, c/o R. White, Plasma Physics Laboratory, Princeton University, P.O. Box 451, Princeton, NJ 08543

118. Theory Department Read File, c/o L. Kovrizhnykh, Lebedev Institute of Physics, Academy of Sciences, 53 Leninsky Prospect, 117924 Moscow, Russia

119. Theory Department Read File, c/o B. B. Kadomtsev, I. V. Kurchatov Institute of Atomic Energy, P.O. Box 3402, 123182 Moscow, Russia

120. Theory Department Read File, c/o T. Kamimura, National Institute for Fusion Studies, Nagoya 464, Japan

121. Theory Department Read File, c/o E. Maschke, Departemente de Recherches sur la Fusion Controlée, CEN/Cadarache, F-13108 Saint-Paul-lez Durance, France

122. Theory Department Read File, c/o D. Düchs, JET Joint Undertaking, Abingdon, Oxfordshire OX14 3EA, United Kingdom

123. Theory Department Read File, c/o R. Briscoe, Culham Laboratory, Abingdon, Oxfordshire OX14 3DB, United Kingdom

124. Theory Department Read File, c/o D. Biskamp, Max-Planck-Institut fur Plasmaphysik, Boltzmannstrasse 2, D-8046 Garching, Federal Republic of Germany

125. Theory Department Read File, c/o T. Takeda, Japan Atomic Energy Research Institute, Tokai Fusion Research Establishment, Tokai-mura, Naka-gun, Ibaraki-ken, Japan

126. Theory Department Read File, c/o J. Greene, General Atomics, P.O. Box 85608, San Diego, CA 92186

127. Theory Department Read File, c/o R. Cohen, Lawrence Livermore National Laboratory, P.O. Box 5511, Livermore, CA 94550

128. Theory Department Read File, c/o R. Gerwin, CTR Division, Los Alamos National Laboratory, P.O. Box 1663, Los Alamos, NM 87545

129. R. Aamodt, Lodestar Research Corporation, 2400 Central Avenue, Boulder, CO 80301

130. K. Appert, Centre de Recherches en Physique des Plasmas, Ecole Polytechnique Fédérale de Lausanne, 21 Avenue des Bains, CH-1007 Lausanne, Switzerland

131. M. Ballico, Max-Planck Institut für Plasmaphysik, Boltzmannstrasse 2, D-8046 Garching, Federal Republic of Germany

132. E. Barbato, Associazione EURATOM-ENEA sulla Fusione, Centro Richerche Energia Frascati, C.P. 65, I-00044 Frascati (Roma), Italy

133. A. Bécoulet, Association EURATOM-CEA sur la Fusion Controlée, Centre d'Etudes Nucléaires de Cadarache, B. P. No. 1, F-13108 Saint Paul lez Durances, France

134. S. Bernabei, Plasma Physics Laboratory, Princeton University, P.O. Box 451, Princeton, NJ 08543

135. A. Bers, Plasma Fusion Center, Massachusetts Institute of Technology, 38-260, Cambridge, MA 02139

136. V. Bhatnagar, JET Joint Undertaking, Abingdon, Oxfordshire OX14 3EA, England 
137. P. T. Bonoli, Plasma Fusion Center, Massachusetts Institute of Technology, 175 Albany St., NW17-115, Cambridge, MA 02139

138. M. Brambilla, Max-Planck Institut für Plasmaphysik, Boltzmannstrasse 2, D-8046 Garching, Federal Republic of Germany

139. Dr. Vincent Chan, General Atomics, P. O. Box 85608, San Diego, CA 92186

140. D. Ehst, Building 205, Argonne National Laboratory, 9700 S. Cass Avenue, Argonne, IL 60538

141. D. Faulconer, Laboratoire de Physique des Plasmas, EURATOM-Etat Belge, Ecole Royale Militaire, Avenue de la Renaissance 30, 1040 Brussels, Belgium

142. D. J. Gambier, JET Joint Undertaking, Abingdon, Oxfordshire OX14 3EA, England

143. S. N. Golovato, Plasma Fusion Center, Massachusetts Institute of Technology, 175 Albany Street, NW17-113, Cambridge, MA 02139

144. W. Ho, SAIC, 10260 Campus Point Drive, San Diego, CA 92121

145. J. Hosea, Plasma Physics Laboratory, Princeton University, P.O. Box 451, Princeton, NJ 08543

146. Kwok Ko, Stanford Linear Accelerator Center, P.O. Box 4349, Stanford, CA 94309

147. C. C. Petty, General Atomics, P.O. Box 85608, San Diego, CA 92186

148. C. K. Phillips, Plasma Physics Laboratory, Princeton University, P.O. Box 451, Princeton, NJ 08543

149. M. Porkolab, Plasma Fusion Center, Massachusetts Institute of Technology, 175 Albany Street, NW17-119, Cambrid.ge, MA 02139

150. R. Prater, General Atomics, P.O. Box 85608, San Diego, CA 92186

151. J. Scharer, Electrical and Computer Engineering Department, 1500 Johnson Drive, University of Wisconsin, Madison, WI 53706

152. P. E. Vandenplas, Laboratoire de Physique des Plasmas, EURATOM-Etat Belge, Ecole Royale Militaire, Avenue de la Renaissance 30, 1040 Brussels, Belgium

153. H. Weitzner, Courant Institute for Mathematical Sciences, New York University, 251 Mercer Street, New York, NY 10001

154. J. R. Wilson, Plasma Physics Laboratory, Princeton University, P.O. Box 451, Princeton, NJ 08543

155-202. Given distribution according to OSTI-4500, Magnetic Fusion Energy (Category Distribution UC-427, Theoretical Plasma Physics) 